\title{
RESEARCH
}

Open Access

\section{NLRP3 inflammasome-mediated microglial pyroptosis is critically involved in the development of post-cardiac arrest brain injury}

Yuan Chang ${ }^{1}$, Juan Zhu' ${ }^{1}$ Di Wang ${ }^{2}$, Hua Li ${ }^{1,3}$, Yihua He ${ }^{1}$, Kewei Liu' ${ }^{1}$ Xiaoqiang Wang ${ }^{1}$, Yuqin Peng ${ }^{1}$, Suyue $\operatorname{Pan}^{1 *+}$ and Kaibin Huang ${ }^{1 *+}$

\begin{abstract}
Background: Brain injury is the leading cause of death and disability in survivors of cardiac arrest, where neuroinflammation is believed to play a pivotal role, but the underlying mechanism remains unclear. Pyroptosis is a pro-inflammatory form of programmed cell death that triggers inflammatory response upon infection or other stimuli. This study aims to understand the role of microglial pyroptosis in post-cardiac arrest brain injury.

Methods: Sprague-Dawley male rats underwent 10-min asphyxial cardiac arrest and cardiopulmonary resuscitation or shamoperation. Flow cytometry analysis, Western blotting, quantitative real-time polymerase chain reaction (qRT-PCR), coimmunoprecipitation, and immunofluorescence were used to evaluate activated microglia and CD11b-positive leukocytes after cardiac arrest and assess inflammasome activation and pyroptosis of specific cellular populations. To further explore the underlying mechanism, MCC950 or Ac-YVAD-cmk was administered to block nod-like receptor family protein 3 (NLRP3) or caspase-1, respectively.

Results: Our results showed that, in a rat model, successful resuscitation from cardiac arrest resulted in microglial pyroptosis and consequential inflammatory infiltration which was mediated by the activation of NLRP3 inflammasome. Targeting NLRP3 and caspase-1, the executor of pyroptosis, with selective inhibitors MCC950 and Ac-YVAD-cmk treatment significantly prevented microglial pyroptosis, reduced infiltration of leukocytes, improved neurologic outcome, and alleviated neuropathological damages after cardiac arrest in modeling rats.
\end{abstract}

Conclusions: This study demonstrates that microglial pyroptosis mediated by NLRP3 inflammasome is critically involved in the pathogenesis of post-cardiac arrest brain injury and provides a new therapeutic strategy.

Keywords: Cardiac arrest, Caspase-1, Microglia, NLRP3, Pyroptosis

\footnotetext{
* Correspondence: pansuyue@smu.edu.cn; hkb@smu.edu.cn

${ }^{\dagger}$ Kaibin Huang and Suyue Pan contributed equally.

'Department of Neurology, Nanfang Hospital, Southern Medical Univerisity,

North Avenue 1838\#, Guangzhou, Guangzhou 510515, China

Full list of author information is available at the end of the article
}

(c) The Author(s). 2020 Open Access This article is licensed under a Creative Commons Attribution 4.0 International License, which permits use, sharing, adaptation, distribution and reproduction in any medium or format, as long as you give appropriate credit to the original author(s) and the source, provide a link to the Creative Commons licence, and indicate if changes were made. The images or other third party material in this article are included in the article's Creative Commons licence, unless indicated otherwise in a credit line to the material. If material is not included in the article's Creative Commons licence and your intended use is not permitted by statutory regulation or exceeds the permitted use, you will need to obtain permission directly from the copyright holder. To view a copy of this licence, visit http://creativecommons.org/licenses/by/4.0/ The Creative Commons Public Domain Dedication waiver (http://creativecommons.org/publicdomain/zero/1.0/) applies to the data made available in this article, unless otherwise stated in a credit line to the data. 


\section{Introduction}

Sudden cardiac arrest is a leading cause of death and disability in China and worldwide [1,2]. Despite the unremitting efforts by health personnel and emergency medical services, as well as increasing awareness about cardiopulmonary resuscitation, only $26 \%$ of adults successfully resuscitated from out-of-hospital cardiac arrest survived to discharge in China [1]. The main reason for the poor prognosis of patients with successful resuscitation is the post-cardiac arrest syndrome, in which brain injury plays a leading role [3]. Although the mechanism underlying post-cardiac arrest brain injury is not fully understood, neuroinflammation has been widely recognized for playing an essential role, as neuroinflammation is characterized by activation of glial cells, an influx of peripheral immune and inflammatory cells, and release of proinflammatory mediators, including cytokines and adhesion molecules [4]. Microglia, as innate immune cells in the brain, may get overactivated to play an initiative role in these inflammatory cascades [4]. Nevertheless, how microglia are primed to fuel the inflammatory response after cardiac arrest remains largely unknown.

Pyroptosis, an emerging form of programmed necrosis, is driven by many cytosolic pattern recognition receptors represented by the nod-like receptor family protein 3 (NLRP3) [5]. Pyroptosis is inherently inflammatory, and it features rapid plasma-membrane rupture and release of pro-inflammatory intracellular contents such as interleukin $1 \beta$ (IL-1 $\beta$ ) and interleukin 18 (IL-18) [6]. Caspase- 1 is the canonical executor of pyroptosis and is also responsible for the processing of gasdermin $\mathrm{D}$ (GSDMD), precursors of IL-1 $\beta$ (pro-IL-1 $\beta$ ) and IL-18 (pro-IL-18). Furthermore, it should be noted that cleavage of GSDMD by the inflammatory caspases including caspase-1 critically drives pyroptosis by releasing the cleaved gasdermin- $\mathrm{N}$ fragment that acts as an intrinsic pyroptosis-inducing factor [7]. Previous studies have demonstrated that NLRP3 is highly expressed in microglia and participates in the assembly of inflammasome by recruiting apoptosis-associated speck-like proteins containing a caspase recruitment domain (ASC) and precursor of caspase-1 (pro-caspase-1), followed by the self-activation of caspase- 1 to trigger cell pyroptosis and consequential excessive inflammation $[8,9]$. The activation of NLRP3 inflammasome has been reported to involve in the pathophysiology of various neurological disorders, such as ischemic stroke [10], intracerebral hemorrhage [11, 12], and traumatic brain injury [13]. However, after cardiac arrest and cardiopulmonary resuscitation, whether the assembly of NLRP3 inflammasome primes microglia and mediates its pyroptosis and consequential neuroinflammation remains unclear. In addition, whether blocking NLRP3 inflammasome is beneficial for alleviating the post-cardiac arrest brain injury remains to be further explored, although it has been shown that blocking the inflammasome is beneficial in cerebral ischemic diseases like stroke in the previous studies [14].

In this study, by using a rat model of asphyxial cardiac arrest and cardiopulmonary resuscitation, we demonstrate that NLRP3 inflammasome-mediated microglial pyroptosis is critically involved in the development of post-cardiac arrest brain injury. Our data showed that cardiac arrest induced microglial pyroptosis and consequential neuroinflammation, mediated by the activation of NLRP3 inflammasome. Targeting NLRP3 and caspase-1 with two highly selective inhibitors MCC950 and Ac-YVAD-cmk dramatically reduced the number of microglia undergoing pyroptosis, the number of infiltrating leukocytes, the elevated level of IL-1 $\beta$ and IL-18, improved neurologic outcome, and alleviated neuronal injury in cardiac arrest-modeling rats. These results may increase our understanding of the mechanism underlying NLRP3 inflammasome-mediated microglia pyroptosis and the therapeutic effect of NLRP3 and caspase-1 inhibitors in the management of post-cardiac arrest brain injury.

\section{Materials and methods Animals}

All animal experiments in this study were approved by the Animal Care and Use Committee of the Nanfang Hospital, Southern Medical University (Guangzhou, China), and adhered to National Institute of Health Guide for the care and use of laboratory animals. Male adult Sprague-Dawley rats (350-400 g) were obtained from the Experimental Animal Center of Southern Medical University and housed in the specific pathogen-free facility at the university under a strict 12-h light/dark cycle with free access to food and water. All efforts were made to minimize the number of animals used in the current study including its suffering.

\section{Rat model of asphyxial cardiac arrest}

The 10-min asphyxial cardiac arrest and cardiopulmonary resuscitation model was performed as described in our previous study [15]. In brief, rats were anesthetized with isoflurane blended with room air (4\% for induction and $2 \%$ for maintenance; RWD, Shenzhen, China), orotracheally intubated with a $14 \mathrm{G}$ cannula (BD, Suzhou, China), and connected to a ventilator (RWD). Intravascular catheters (PE50; Smiths Medical, Ashford, UK) were inserted into the right femoral artery and vein for dynamic blood pressure monitoring and drug delivery, respectively. After 10-min of stabilization, rats were chemically paralyzed by IV Vecuronium $(2 \mathrm{mg} / \mathrm{kg})$, and then the ventilator was disconnected for $10 \mathrm{~min}$, typically leading to circulatory arrest in $5 \mathrm{~min}$, which was 
characterized by cessation of arterial pulse and a decrease in mean arterial pressure (MAP) below $20 \mathrm{mmHg}$. At the end of 10-min asphyxia, cardiopulmonary resuscitation was initiated by effective ventilation with 100\% oxygen as the inspired gas and intravenous administration of epinephrine $(0.01 \mathrm{mg} / \mathrm{kg})$. Concurrently, finger chest compressions were conducted by the operator at a rate of about 200 compressions per minute. Additional doses of epinephrine $(0.02 \mathrm{mg} / \mathrm{kg})$ were given at 2 -min intervals until return of spontaneous circulation (ROSC) was achieved. ROSC was defined as an increase in MAP beyond $60 \mathrm{mmHg}$ lasting at least $10 \mathrm{~min}$. Rats that failed to ROSC within $5 \mathrm{~min}$ or were unable to be weaned from ventilator after 1 -h observation were excluded from the continuing experiments.

During the whole procedure, core temperature was monitored by a rectal temperature probe (RC-4; Elitech, Xuzhou, China) and maintained at $37.0 \pm 0.5{ }^{\circ} \mathrm{C}$ with a temperature feedback system (RWD). After spontaneous respiration recovery, rats were weaned from ventilator and extubated. Afterward, all catheters were removed and surgical wounds were sutured. At the end of each experimental period, rats were returned to their cages with easily accessible food and water and were observed in a room of constant temperature $\left(22{ }^{\circ} \mathrm{C}\right)$. Saline $(20 \mathrm{~mL} / \mathrm{kg})$ was injected daily to rats until they were able to feed themselves.

\section{Study protocol}

Rats were randomly assigned to appropriate groups for different experimental purposes by using random number tables (Fig. 1). MCC950 (MedChem Express, Monmouth, NJ, USA) was dissolved in sterile saline at a concentration of $2 \mathrm{mg} / \mathrm{mL}(4.94 \mathrm{mM})$, and $10 \mathrm{mg} / \mathrm{kg}$ $(24.72 \mu \mathrm{mol} / \mathrm{kg})$ was administered intraperitoneally at 10 min after ROSC [16, 17], while rats in the vehicle group received the equivalent volume of saline. For rats utilized to assess 7-day survival rate and neurologic outcome, MCC950 was given once daily for 6 days [18]. Ac-YVADcmk (Sigma-Aldrich, St. Louis, MO) was dissolved in dimethyl sulfoxide (DMSO) and diluted in saline to a concentration of $100 \mathrm{ng} / \mu \mathrm{L} \quad(0.18 \mathrm{mM}$ containing $0.6 \%$ DMSO), and was intracerebroventricularly (i.c.v.) injected into the right lateral ventricle of fixed rats with a loading dose of $400 \mathrm{ng} / \mathrm{rat}$ at 25-30 min before the surgeries [19], whereas rats in the vehicle group received equal volume of DMSO and saline only. Rats that underwent all procedures except asphyxial cardiac arrest and cardiopulmonary resuscitation were used as sham control.

The present study included 3 parts (Fig. 1b). In part 1, the occurrence of pyroptosis in cardiac arrest model was explored using flow cytometry, Western blotting, quantitative real-time polymerase chain reaction (qRT-PCR), co-immunoprecipitation, and histological evaluation, in which 44 rats that successfully resuscitated were implicated. In part 2, 44 rats with successful resuscitation ( $n=22$ each for MCC950 and vehicle groups) were followed up to assess survival rate, neurologic outcome, short-term memory and learning ability, and histological impairment. The other 46 rats achieving ROSC $(n=5$ or 6 for each group) were used to illustrate the protective effect of MCC950 in the acute phase (12 h) by detecting pyroptosis and associated molecular expression. In part 3 , rats were randomly allocated to receive either AcYVAD-cmk or an equal volume of DMSO and saline at about $30 \mathrm{~min}$ before surgery, and the follow-up experiments were similar to that of part 2 .

\section{Flow cytometry}

The procedure of flow cytometry was established as described previously with some adjustments [13]. Briefly, at 48 -h post-surgery, all rats were anesthetized with overdose isoflurane and then perfused transcardially with ice-cold phosphate-buffered saline (PBS). The bilateral cerebral cortex and hippocampus were mechanically isolated into a single-cell suspension by passage through a 40- $\mu \mathrm{m}$ cell strainer (Falcon, Madison, WI) on ice and placed in ice-cold PBS. Afterward, cells were labeled with a FAM-FLICA assay (Immunochemistry Technologies, Bloomington, $\mathrm{MN}$ ) to detect the caspase-1 activity, in which the FLICA reagent known as FAM-YVADFMK forms an irreversible covalent bond with the fluoromethyl ketone (FMK) to the cleaved caspase-1. The carboxyfluorescein (FAM) optimally excites at $488-492$ $\mathrm{nm}$ and has a peak emission at $515-535 \mathrm{~nm}$. Cell viability was assessed via the LIVE/DEAD Fixable Near-IR Dead Cell Stain (L10119, $1 \mu \mathrm{L} / \mathrm{mL}$, Life Technologies) following the manufacturer's instructions, which is featured as membrane-impermeable and combines with amine residues of membrane proteins when the cell membrane is integrated leading to a low level of fluorescence. The LIVE/DEAD Fixable Near-IR Dead Cell Stain is allowed to enter the dying cells with increased membrane permeability, thereby causing the enhancement of fluorescence in cells. After a non-specific block with CD16/CD32 antibody, the cells were further followed with surface markers CD45 Alexa 647 antibody (202212, $1.25 \mu \mathrm{g} / \mathrm{mL}$, BioLegend) and CD11b v450 antibody (562108, $0.2 \mathrm{mg} / \mathrm{mL}$, BD Horizon). Gates were established using antibody isotype controls (provided by manufacturers) and fluorescence minus one controls. The samples were acquired on LSRII/Fortessa flow cytometer (BD Biosciences, Heidelberg, Germany). Finally, the resulting flow cytometry files were analyzed with Flowjo V10 software.

\section{Western blotting}

Western blotting was routinely performed as previously reported [20]. Mouse anti- $\beta$-actin (1:10,000, Proteintech, 


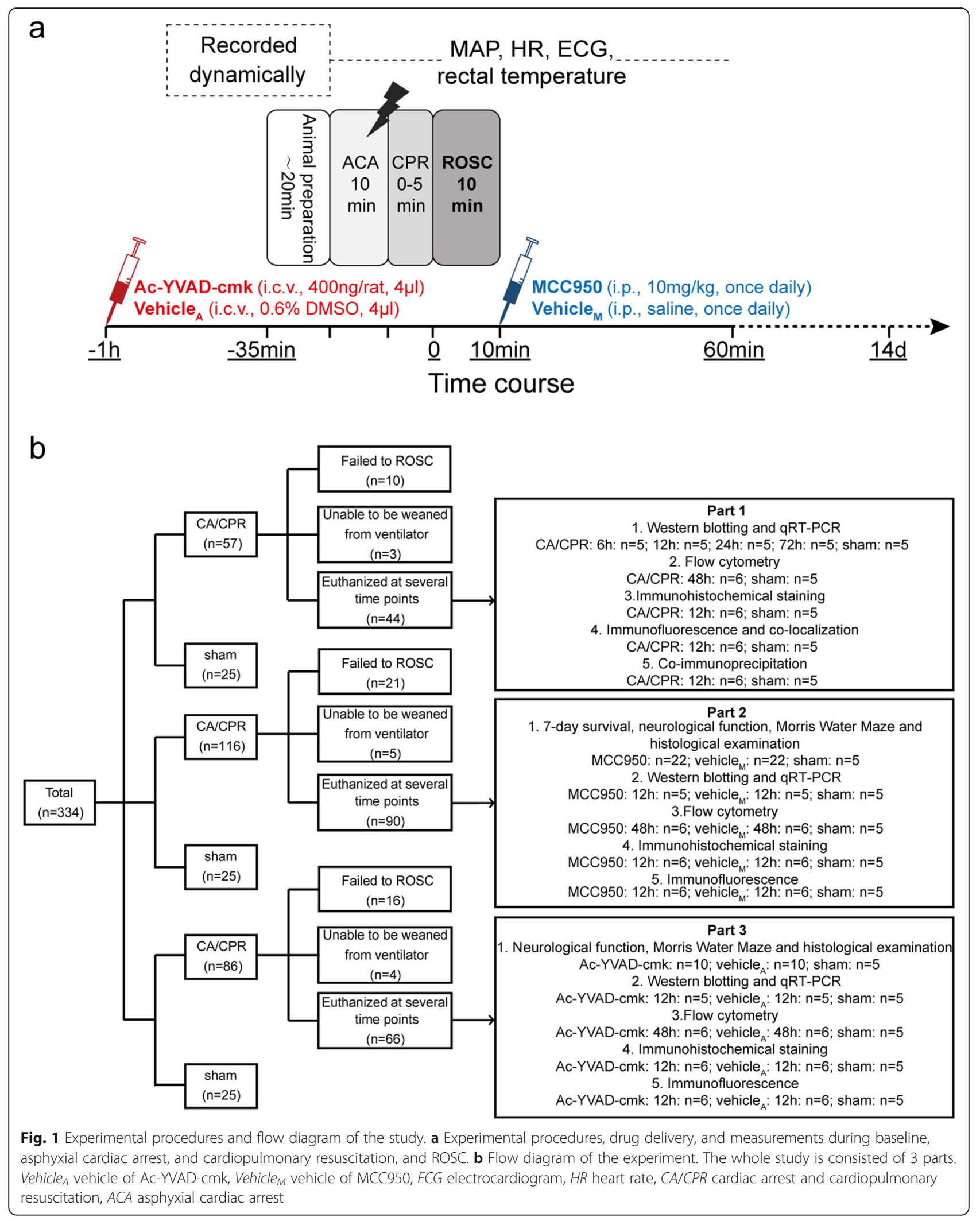


Chicago, IL), rabbit anti-NLRP3 (1:500, Novus Biologicals, CO, USA), rabbit anti-caspase-1 (1:500, Proteintech), mouse anti-ASC (1:500, Santa Cruz, CA, USA), rabbit anti-GSDMD (1:1000, CST, Danvers, USA), mouse anti-pro-IL-1 $\beta$ (1:1000, Proteintech), rabbit anticleaved IL-1 $\beta$ (1:1000, Novus Biologicals), rabbit anti-proIL-18 (1:1000, Proteintech), rabbit anti-cleaved IL-18 (1:300, Bioss, Beijing, China), and rabbit anti-caspase-11 (1:1000, Novus Biologicals) were used. The densities of protein blots were quantified by using ImageJ software $(\mathrm{NIH}$, Bethesda, MD) and normalized to the level of $\beta$-actin.

\section{Measurement of gene expression}

The mRNA levels of NLRP3, ASC, caspase-1, GSDMD, IL-1 $\beta$, IL-18, caspase-11, and GADPH were routinely measured by qRT-PCR [20]. Relative changes of mRNA expressions were normalized to levels of GADPH.

\section{Survival study and neurologic function evaluation}

Rats subjected to cardiac arrest modeling were followed up to 7 days, and survival rate was recorded daily. A previously validated scale of neurologic deficit score (NDS) ranging from 0 to 80 was utilized to assess the neurologic outcome at 24, 48, $72 \mathrm{~h}$ and 7 days after ROSC, which was performed by two investigators blinded to animal grouping [21]. The total NDS scale consisted of 7 components: general behavioral deficit, brain-stem function, motor assessment, sensory assessment, motor behavior, behavior, and seizures, in which 80 was considered normal, whereas 0 represented brain death.

\section{Behavioral testing}

The Morris water maze was carried out to evaluate short-term spatial learning and memory as described previously [22]. The water maze apparatus is a circular tank filled with $22-24{ }^{\circ} \mathrm{C}$ opaque water rendered by black food pigment to a depth of $25 \mathrm{~cm}$ (divided into four quadrants, called Q1, Q2, Q3, Q4) and containing a hidden platform which is submerged 2-3 $\mathrm{cm}$ below the water surface and not visible to the rats at the fixed location of Q3. Firstly, the rats were trained to search for the platform on post-surgery day 9-12 at a frequency of four trials/day, orienting by referencing 3 external cues surrounding the tank. If the rats did not find the platform in $60 \mathrm{~s}$, they were manually placed on it for $30 \mathrm{~s}$. Rats' movements were tracked by TSE VideoMot2 video tracking system (TSE Systems GmbH, Bad Homburg, Germany) to record the path and time taken to escape from 4 randomly assigned locations. The latency time required to locate the hidden platform was compared among groups. After the hidden platform training, the platform was removed in the probe test, and the rats had $60 \mathrm{~s}$ to search for the pool. The percentage of the total time that rats spent in the target quadrant and the number of platform location crossings were analyzed.

\section{Histological examination}

Rats were euthanized after the Morris water maze experiment or predefined points (Fig. 1b), and 4- $\mu \mathrm{m}$-thick coronal brain sections located at $3.5 \mathrm{~mm}$ posterior to bregma were obtained (Leica CM1800; Leica Microsystems $\mathrm{GmbH}$, Heidelberg, Germany). For detecting neuronal loss, brain sections were stained with cresyl violet (Beyotime, Shanghai, China) and observed under microscope (Olympus, Tokyo, Japan). Viable neurons in the hippocampal CA1 region were those characterized as visible nucleus and intact cytoplasm with discernable and rich Nissl staining, while those with shrunken cell bodies surrounded by empty space were regarded as dead neurons.

Immunohistochemistry was conducted by incubation of sections with antibodies against neuronal nuclei (NeuN; CST) for detection of neuron, microtubuleassociated protein 2 (MAP2; Sigma-Aldrich) for dendrite, glial fibrillary acidic protein (GFAP; Abcam, Cambridge, UK) for astrocyte, ionized calcium-binding adapter molecule-1 (Iba-1; Wako, Osaka, Japan) for all microglia, and CD68 (Abcam) for activated microglia. In each brain section, 3 slide-fields were randomly examined. The relative intensity area of MAP-2 immunoreactivity in hippocampus and cortex, and the number of cells with immunoreactivity of other above markers were quantified by an observer blinded to experimental grouping using Image J software (NIH).

For the immunofluorescence method, brain sections were incubated with the following primary antibodies: goat anti-NLRP3 (Abcam), mouse anti-ASC (Santa Cruz), mouse anti-caspase-1 (Santa Cruz), rabbit antiNLRP3 (Novus), rabbit anti-caspase-1 p20 (Abcam), rabbit anti-Iba-1 (Abcam) for microglia, rabbit antiGFAP (Abcam) for astrocytes, rabbit anti-NeuN (CST) for Neurons, rabbit anti-CD31 (Abcam) for endothelial cells, rabbit anti-Neuron Glial Antigen 2 (NG2; Proteintech) for oligodendrocyte precursor cells, and rabbit anti-oligodendrocyte lineage transcription factor 2 (Olig2; Abcam) for oligodendrocytes. The sections were then washed and detected with appropriate Alexa Fluor dye of secondary antibodies followed by counterstained with DAPI in the dark. Afterward, immunofluorescent signaling was observed with a confocal microscope (FluoView; Olympus). Digital images were recorded and analyzed using Image (NIH).

\section{Co-immunoprecipitation}

This test was performed according to the manual for Protein A/G Magnetic Beads (Bimake, Houston, USA). 
In brief, cerebral cortex and hippocampus were lysed in lysis buffer containing phenylmethylsulfonyl fluoride (PMSF) for IP (Genstar). Thereafter, cytoplasmic protein extraction was performed according to the manufacturer's instructions (Beyotime). After centrifuged for 15 min at $14,000 \mathrm{~g}, 4{ }^{\circ} \mathrm{C}$, the supernatant was obtained and quantified, of which the portion containing $500 \mu \mathrm{g}$ protein was immunoprecipitated with $1 \mu \mathrm{g}$ rabbit antiNLRP3 (Abcam) or mouse anti-ASC (Santa Cruz) under rotation overnight at $4{ }^{\circ} \mathrm{C}$. After that, immune complexes were collected, then it was incubated with the added $30 \mu \mathrm{L}$ protein $\mathrm{A} / \mathrm{G}$ magnetic beads under rotation for another $30 \mathrm{~min}$ at room temperature and separated magnetically. And then the beads were washed 5 times with a pH 7.5 wash buffer containing $50 \mathrm{mM}$ Tris, 150 $\mathrm{mM} \mathrm{NaCl}, 0.1 \% \mathrm{NP} 40$, resuspended in $40 \mu \mathrm{L} 1 \times$ loading buffer, and heated at $100{ }^{\circ} \mathrm{C}$ for $5 \mathrm{~min}$. At the end, the protein complexes were subjected to Western blotting as described above, and the employed antibodies were as follows: rabbit anti-NLRP3 (Novus Biologicals), mouse anti-ASC (Santa Cruz), mouse anti-caspase-1 (Santa Cruz), and rabbit anti-caspase-1 (Proteintech). Whole tissue lysate prepared for IP was used as an input and homophytic IgG as the negative control. ImageJ was employed to calculate the protein ratio of NLRP3associated ASC and pro-caspase-1 or ASC-associated NLRP3 and pro-caspase-1.

\section{Statistical analysis}

All data were presented as means \pm SD or medians and 25th to 75th percentiles (NDSs). Continuous data were analyzed with two-sided Student's $t$ test, one-way ANOVA followed by Tukey's post hoc multiple comparison tests, or as indicated. The difference in survival rate was measured by Kaplan-Meier analysis with logrank test. NDSs were compared with Mann-Whitney $U$ test. Physiological variables and the data of escape latency in the water maze training were analyzed with repeated-measures ANOVA comprising treatment groups, time points, and treatment $\times$ time points interaction, followed by Tukey's post hoc multiple comparison tests. SPSS 20.0 (IBM, Armonk, NY) and GraphPad Prism 6.0 (GraphPad, La Jolla, CA) were used for statistical analyses. $P<0.05$ was considered statistically significant.

\section{Results}

\section{Cardiac arrest leads to microglia activation and leukocytes infiltration in the brain}

Rats undergoing10-min asphyxial cardiac arrest and cardiopulmonary resuscitation modeling $(n=259)$ or sham operation $(n=75)$ were randomly assigned to appropriate groups for different experimental purposes (Fig. 1). We first sought to examine the influence of cardiac arrest on the status of microglia. At $48 \mathrm{~h}$ post-surgery, single-cell suspension dissociated from cortical and hippocampal brain tissues were prepared to differentiate the populations of resident microglia and infiltrating leukocytes by flow cytometry labeling with CD 45 and CD11b $[23,24]$. As reported [25, 26], activated microglia possess a higher level of CD45 than resting microglia, while infiltrating leukocytes own the highest amounts of CD45. Consequently, CD11b-positive cells including infiltrating myeloid-lineage leukocytes and microglia could be classified as 3 populations: low CD45 population (CD45 low, $\left.\mathrm{CD}_{11} \mathrm{~b}^{+}\right)$, intermediate $\mathrm{CD} 45$ population $\left(\mathrm{CD} 45_{\text {int }}\right.$, $\left.\mathrm{CD}_{11 \mathrm{~b}^{+}}\right)$, and high CD45 population (CD45 high, $\mathrm{CD} 1 \mathrm{~b}^{+}$), and they may represent resting microglia, activated microglia, and invading leukocytes, respectively (Fig. 2a, b). Cells in the sham-operated hippocampus expressed a low level of CD45 $\left(\mathrm{CD} 45_{\text {low }}, \mathrm{CD} 11 \mathrm{~b}^{+}\right)$, manifesting as tight cell clusters on the graph with very few cells in the CD45 int and CD45 high range. As a contrast, cells in the post-cardiac arrest hippocampus revealed much higher expression of both CD45 and CD11b, reflected as looser cell clusters on the graph with many more cells in the CD45 $5_{\text {int }}$ and CD45 high range. Similar results were observed in cells from cortical brain tissues. It indicates a large number of microglia shifts from an immuno-surveilling state to an activated state after cardiac arrest, accompanied by massive exudation of myeloid-lineage leukocytes in the post-cardiac arrest brain tissues.

\section{Cardiac arrest triggers microglial pyroptosis and an} increase of caspase- 1 activity in the activated microglia We next investigated whether the activated microglia were correlated with pyroptosis and promoted the inflammatory response after cardiac arrest. Flow cytometry was conducted to measure the cell viability and caspase- 1 activity in microglia, which were labeled with LIVE/DEAD Fixable Near-IR Dead Cell Stain fluorescent probe and FAM-FLICA fluorescent probe, respectively. The $\mathrm{CD}_{11 \mathrm{~b}^{+}}$cells were at first gated into $\mathrm{CD} 45_{\text {low }} \mathrm{CD} 11 \mathrm{~b}^{+}, \mathrm{CD} 45_{\text {int }} \mathrm{CD} 11 \mathrm{~b}^{+}$and $\mathrm{CD} 45_{\text {high }} \mathrm{CD} 11 \mathrm{~b}^{+}$by $\mathrm{CD} 45$ labeling, as described above. Then, all microglia including resting $\left(\mathrm{CD} 45_{\text {low }}, \mathrm{CD} 11 \mathrm{~b}^{+}\right)$and activated microglia $\left(\mathrm{CD} 45_{\text {int }}\right.$, $\mathrm{CD}_{11 \mathrm{~b}^{+}}$) were plotted using the two kinds of fluorescent probes to establish a four-quadrant gate (Fig.

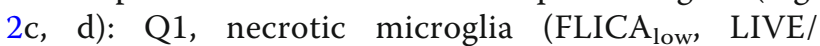
DEAD $\left._{\text {high }}\right) ; \quad$ Q2, pyroptotic microglia $\left(\right.$ FLICA $_{\text {high }}$, LIVE/DEAD high $_{\text {h }}$; Q3, live microglia expressing caspase-1 (FLICA high $_{\text {, LIVE/DEAD }}$ low $) ; \quad$ Q4, live microglia without expression of caspase-1 $\left(\mathrm{FLICA}_{\text {low }}\right.$, LIVE/DEAD low $_{\text {lout }}$ ) [13].

As illustrated, the number of pyroptotic microglia and live microglia expressing caspase- 1 in the post-cardiac 


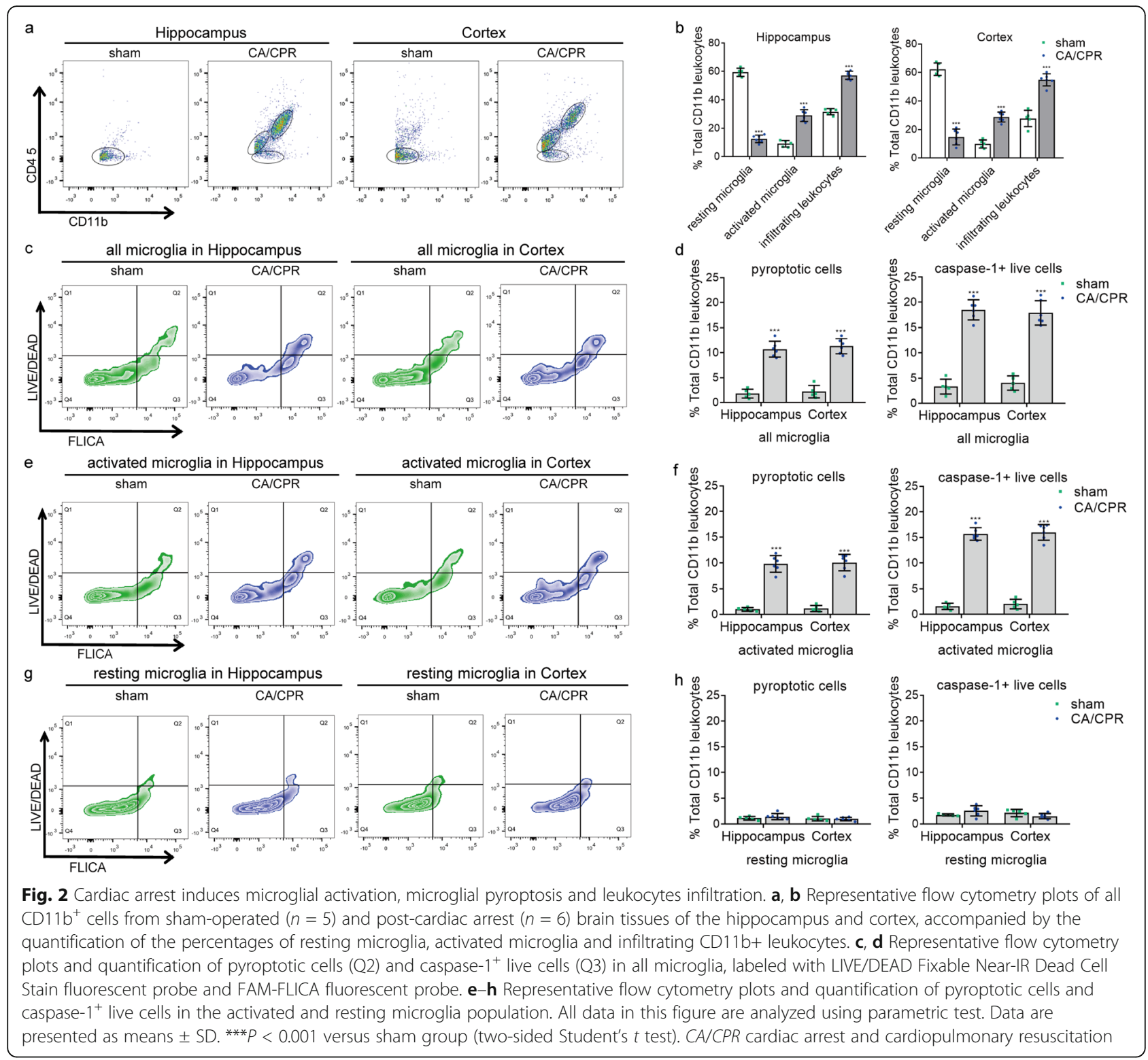

arrest brain tissues (hippocampus and cortex) was significantly higher than that in the sham-operated brain tissues (Fig. 2c, d). Further analysis revealed that the number of cells undergoing pyroptosis and live cells expressing caspase- 1 was predominantly increased in the

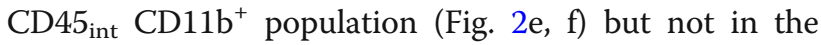
$\mathrm{CD} 45_{\text {low }} \mathrm{CD} 11 \mathrm{~b}^{+}$population (Fig. $2 \mathrm{~g}, \mathrm{~h}$ ), indicating that the activated microglia population was the primary source of pyroptotic microglia and cells with elevated caspase-1 activity after cardiac arrest. These results imply that cardiac arrest triggers microglial pyroptosis and an increase of caspase-1 activity in the activated microglia, and the latter may also subsequently undergo pyroptosis.

\section{Cardiac arrest induces NLRP3 inflammasome activation in microglia}

Inflammasome assembling with NLRP3 and caspase-1 is considered to be the major signaling molecule that promotes the pyroptosis in microglia [27]. Therefore, we tested the activation of NLRP3 inflammasome after cardiac arrest, aiming to provide potential targets for mediating microglial pyroptosis. Results from qRT-PCR showed that the mRNA level of NLRP3 was significantly upregulated after cardiac arrest, with a peak at the 12th hour (Fig. 3a). Consistently, the protein level of NLRP3 was elevated at 6 to $24 \mathrm{~h}$ after cardiac arrest (Fig. 3b, c). The mRNA level of caspase- 1 and the protein level of pro-caspase-1 were not altered for the rats undergoing 


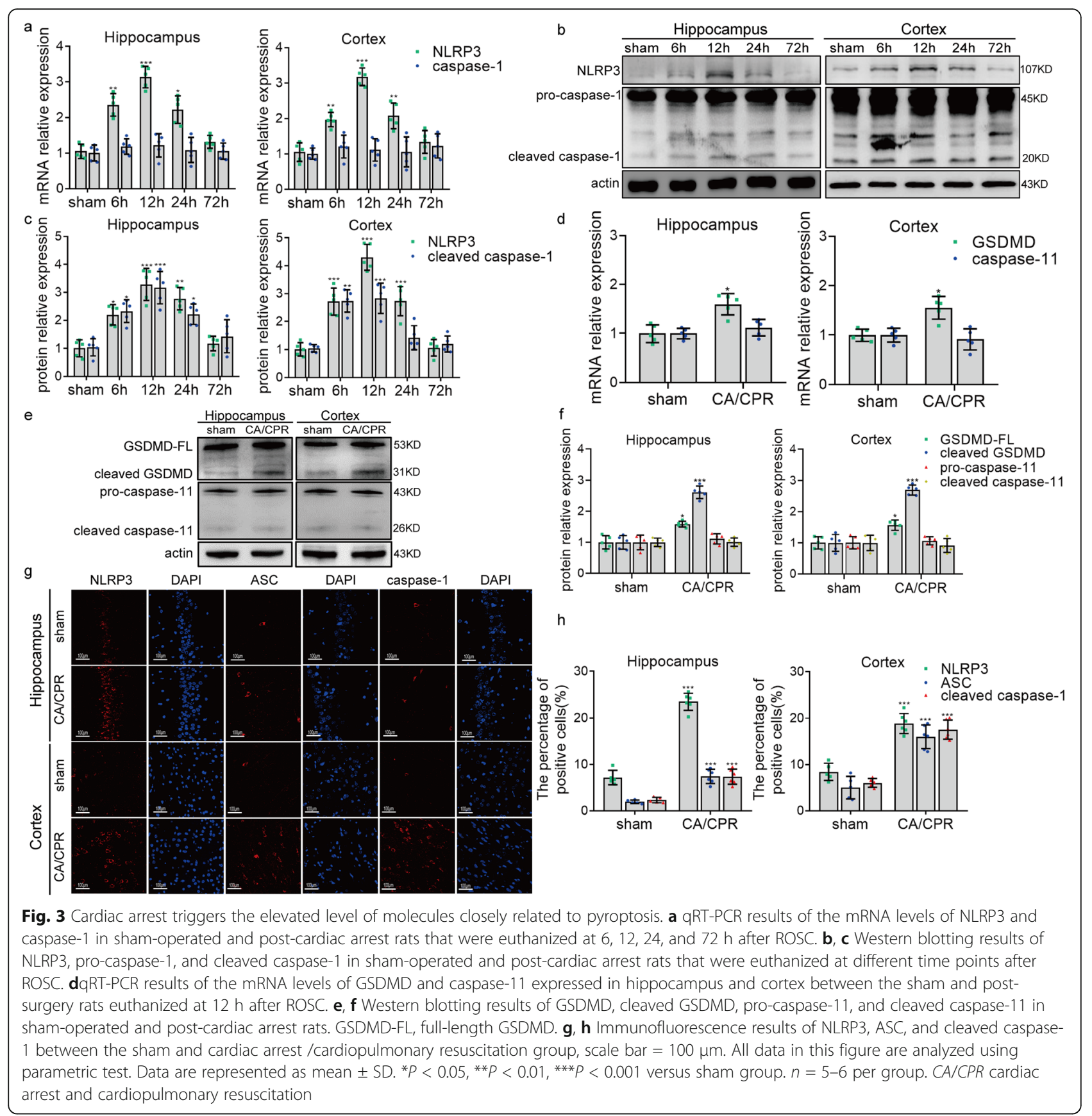

cardiac arrest compared to sham operation (Fig. 3a-c). However, the protein level of cleaved caspase-1, namely the active form of capase-1, was increased at 6 to $24 \mathrm{~h}$ in the post-cardiac arrest brain (Fig. 3b, c), indicating that the pro-caspase- 1 underwent autocatalysis and activation after the cardiac arrest. In addition, the mRNA and protein levels of GSDMD were further evaluated via qRTPCR and Western blotting to prove that the cleavage of caspase-1 here was involved in pyroptosis but not apoptosis [28]. As expected, it was observed that the protein level of cleaved GSDMD was markedly increased in the post-cardiac arrest brain, with the elevated mRNA and protein levels of GSDMD (Fig. 3d-f), which implies that caspase- 1 is cleaved and activated after cardiac arrest, leading to the downstream cleavage of GSDMD and pyroptosis. We also assessed the level of caspase-11, in order to exclude the effect of caspase-11-mediated noncanonical inflammasome activation on the cleavage of GSDMD and pyroptosis [29]. It was found that there was no significant change in the protein levels of precursors of caspase-11 (pro-caspase-11) and cleaved caspase11 of the rats in the cardiac arrest group compared to 
that in the sham group (Fig. 3d-f), indicating that it is the canonical NLRP3 inflammasome that mediates the microglia pyroptosis in the rats after cardiac arrest, and caspase-1 but not caspase-11 is involved in the process. The elevated expression of NLRP3 and cleaved caspase1 were further confirmed by immunofluorescent staining at $12 \mathrm{~h}$ after cardiac arrest, along with ASC, another key molecule involved in the assembly of inflammasome (Fig. 3g, h).

To further validate whether the assembly of inflammasome principally occurred in microglia after cardiac arrest, immunofluorescent co-localization was performed. Using confocal microscopy, we observed that NLRP3 and caspase-1 were mainly co-localized in Iba-1-positive cells but not other types of cells such as neuron, astrocytes, endothelial cells, oligodendrocyte, or oligodendrocyte precursor cells to form the inflammasome (Fig. 4a-d). It should be emphasized that since Iba-1 is not a highly specific marker, the Iba-1-positive cells, in addition to identifying microglia, may also represent a small number of invading monocytic lineage cells like macrophages. The association of NLRP3, ASC, and caspase-1 represents the

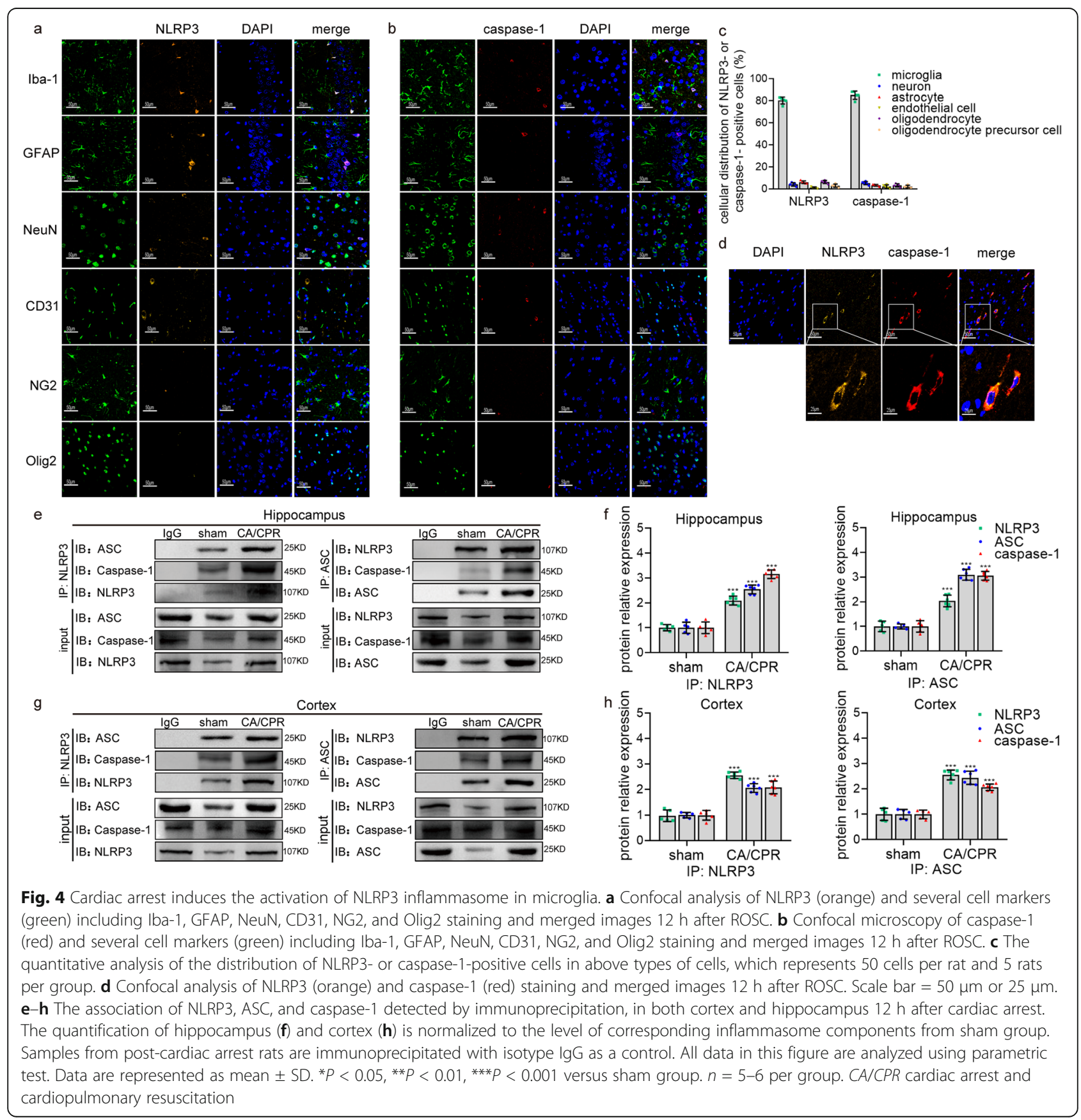


intracellular activation of the inflammasome [27]. As shown by the co-immunoprecipitation (Fig. 4e-h), pairwise interactions among NLRP3, ASC, and caspase-1 were observed in both the hippocampus and cortex tissues at $12 \mathrm{~h}$ after cardiac arrest. Our quantitative analysis showed that the assembly of NLRP3 inflammasomes in microglia of postsurgery rats was significantly increased, featuring in the increment in the absolute amount. Interestingly, we found that there was a small amount of activated inflammasomes in sham group, which could be attributed to the effect of isoflurane anesthesia [30]. Therefore, we speculate that the combination of isoflurane and cardiac arrest in our study may lead to additional inflammasome activation over that seen in cardiac arrest without anesthesia. Taken together, these findings imply that cardiac arrest activates the assembly of NLRP3 inflammasome in microglia, which leads to the self-cleavage of caspase- 1 and triggers microglial pyroptosis.

\section{Targeting NLRP3 with MCC950 prevents microglial pyroptosis and consequential neuroinflammation after cardiac arrest}

To further test the role of NLRP3 inflammasome and correlated microglia pyroptosis in post-cardiac arrest brain injury, we targeted the NLRP3 with a highly selective inhibitor MCC950 [16]. At 10 min after ROSC from cardiac arrest, the rats were randomized to receive daily once MCC950 or vehicle until euthanasia (Fig. 1). The body weight, the time from asphyxia to cardiac arrest, the time required for ROSC, and epinephrine usage were all comparable between the MCC950 and vehicle groups
(Table 1). There were also no statistical differences in physiological variables, including MAP, heart rate, and rectal temperature at baseline or after ROSC between these two groups (Table 1).

Given the direct bioactivity of MCC950 on NLRP3, we first examined the influence of MCC950 on the assembly of NLRP3 inflammasome, the cleavage of caspase- 1 and GSDMD, and the maturation of IL-1 $\beta$ and IL-18. The results from qRT-PCR and Western blotting showed that MCC950 significantly suppressed the levels of NLRP3, ASC, IL-1 $\beta$, and IL-18 at $12 \mathrm{~h}$ after cardiac arrest, in both hippocampus and cortex (Fig. 5a-c). Also, MCC950 markedly inhibited the activity of cleaved caspase- 1 but not the level of pro-caspase-1, suggesting that MCC950 suppresses the activation but not the generation of caspase-1. Besides, we found the similar result that MCC950 could significantly inhibit the activation of GSDMD and reverse the increased level of cleaved GSDMD after surgery, with no effect on the level of fulllength GSDMD (Fig. 5a-c). Consistently, less NLRP3positive, ASC-positive, and cleaved caspase-1-positive cells were observed in the MCC950-treated group (Fig. $5 \mathrm{~d}, \mathrm{e})$.

We then examined whether MCC950 prevents microglial pyroptosis and the increased caspase- 1 activity in activated microglia caused by cardiac arrest. In consistent with the above results, a reduced number of live cells expressing caspase- 1 and pyroptotic cells were observed in the MCC 950 but not the vehicle groups at $48 \mathrm{~h}$ after cardiac arrest (Fig. 6a, b). MCC950 treatment significantly reduced the number of pyroptotic cells and live

Table 1 Parameters of the Vehicle ${ }_{M}$ and MCC950 groups at baseline and during post-cardiac arrest care after ROSC

\begin{tabular}{|c|c|c|c|}
\hline Parameters & Time points & Vehicle $_{M}$ & MCC950 \\
\hline Body weight, $g$ & Baseline & $377 \pm 18$ & $384 \pm 20$ \\
\hline Time from asphyxia to cardiac arrest, s & Baseline & $214.0 \pm 21$ & $210.9 \pm 17$ \\
\hline Time required for $\mathrm{ROSC}$, $\mathrm{s}$ & Baseline & $90.77 \pm 24$ & $93.78 \pm 27$ \\
\hline Total dose of epinephrine, $\mu \mathrm{g}$ & Baseline & $13.2 \pm 3.1$ & $12.0 \pm 3.4$ \\
\hline \multirow[t]{4}{*}{ Mean arterial pressure, $\mathrm{mmHg}$} & Baseline & $122 \pm 8$ & $120 \pm 11$ \\
\hline & $10 \mathrm{~min}$ & $113 \pm 7$ & $110 \pm 9$ \\
\hline & $30 \mathrm{~min}$ & $88 \pm 10$ & $91 \pm 7$ \\
\hline & $60 \mathrm{~min}$ & $85 \pm 7$ & $81 \pm 9$ \\
\hline \multirow[t]{4}{*}{ Heart rate, beats/min } & Baseline & $400 \pm 11$ & $399 \pm 9$ \\
\hline & $10 \mathrm{~min}$ & $419 \pm 17$ & $424 \pm 20$ \\
\hline & $30 \mathrm{~min}$ & $364 \pm 17$ & $370 \pm 16$ \\
\hline & $60 \mathrm{~min}$ & $375 \pm 14$ & $380 \pm 9$ \\
\hline \multirow[t]{4}{*}{ Rectal temperature, ${ }^{\circ} \mathrm{C}$} & Baseline & $37.1 \pm 0.3$ & $37.0 \pm 0.2$ \\
\hline & $10 \mathrm{~min}$ & $37.1 \pm 0.2$ & $36.8 \pm 0.3$ \\
\hline & $30 \mathrm{~min}$ & $37.0 \pm 0.3$ & $36.9 \pm 0.2$ \\
\hline & $60 \mathrm{~min}$ & $37.0 \pm 0.2$ & $36.9 \pm 0.3$ \\
\hline
\end{tabular}

Physiological variables are measured at baseline and at 10,30, and $60 \mathrm{~min}$ after ROSC. The values are expressed as mean \pm SD. Vehicle ${ }_{M}$ vehicle of MCC950 


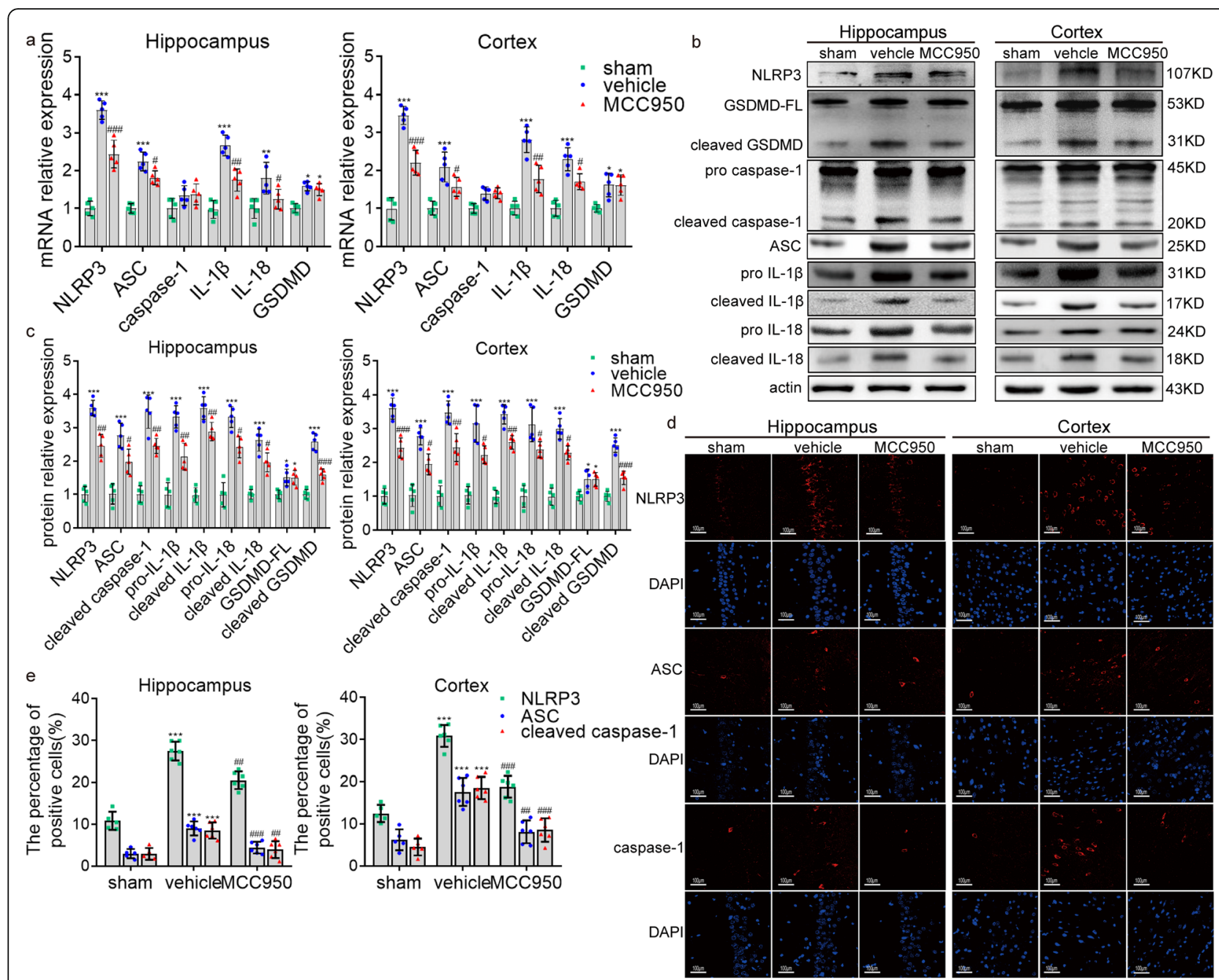

Fig. 5 The effect of MCC950 on the level of molecules associated with pyroptosis. a-c qRT-PCR and Western blotting results showing the mRNA levels (a) and protein levels (b, c) of NLRP3, ASC, caspase-1, GSDMD, IL-1 $\beta$, and IL-18 among the sham, vehicle, and MCC950 groups. GSDMD-FL, full-length GSDMD. $\mathbf{d}$, e Immunofluorescence staining results showing the protein expression of NLRP3, ASC, and cleaved caspase-1 among the sham, vehicle, and MCC950 groups. All data in this figure are analyzed using parametric test. Data are presented as mean \pm SD. Statistical significances are determined with one-way ANOVA followed by Tukey's post hoc test. ${ }^{*} P<0.05,{ }^{* *} P<0.01$, ${ }^{* * *} P<0.001$ versus sham; $\# P<0.05$, $\# \#>0.01, \# \# P<0.001$ versus vehicle. $n=5-6$ per group

cells expressing caspase- 1 compared to the vehicle group in the $\mathrm{CD} 45_{\text {int }} \mathrm{CD}_{11 \mathrm{~b}^{+}}$population (Fig. 6a, b). However, there was no difference in the number of pyroptotic and caspase-1-positive cells between the MCC950-treated and the vehicle-treated groups in the $\mathrm{CD} 45_{\text {low }} \mathrm{CD} 11 \mathrm{~b}^{+}$ population (Fig. 6a, b). Moreover, the delivery of MCC950 led to a significant reduction in the number of CD $45_{\text {high }} \mathrm{CD}_{11 \mathrm{~b}^{+}}$cells (Fig. 6c, d) accompanied by the inhibition of IL- $1 \beta$ and IL-18, suggesting that the intervention of NLRP3 by MCC950 prevents the post-cardiac arrest inflammatory response. Strikingly, MCC950 inhibited not only the mature IL-1 $\beta$ and IL- 18 but also their precursors, which could be attributed to the overall suppression of inflammatory response and thus reduced the transcription of these two cytokines by nf-kb [31-33].
Therefore, our findings provide evidence that targeting NLRP3 by MCC950 suppresses the occurrence of microglial pyroptosis and consequential inflammatory response after cardiac arrest.

\section{Targeting NLRP3 with MCC950 improves survival and neurologic outcome after cardiac arrest}

We then explored whether the suppression of microglia pyroptosis and consequential inflammatory response improved outcomes by targeting NLRP3 with MCC950 in cardiac arrest modeling rats. We found that the 7-day survival rate in the MCC950 group (72.7\%, 16 of 22) was significantly higher than that in the vehicle group (40.9\%, 9 of 22) (Fig. 7a). In addition, we used the NDS to assess the neurologic function after cardiac arrest and 


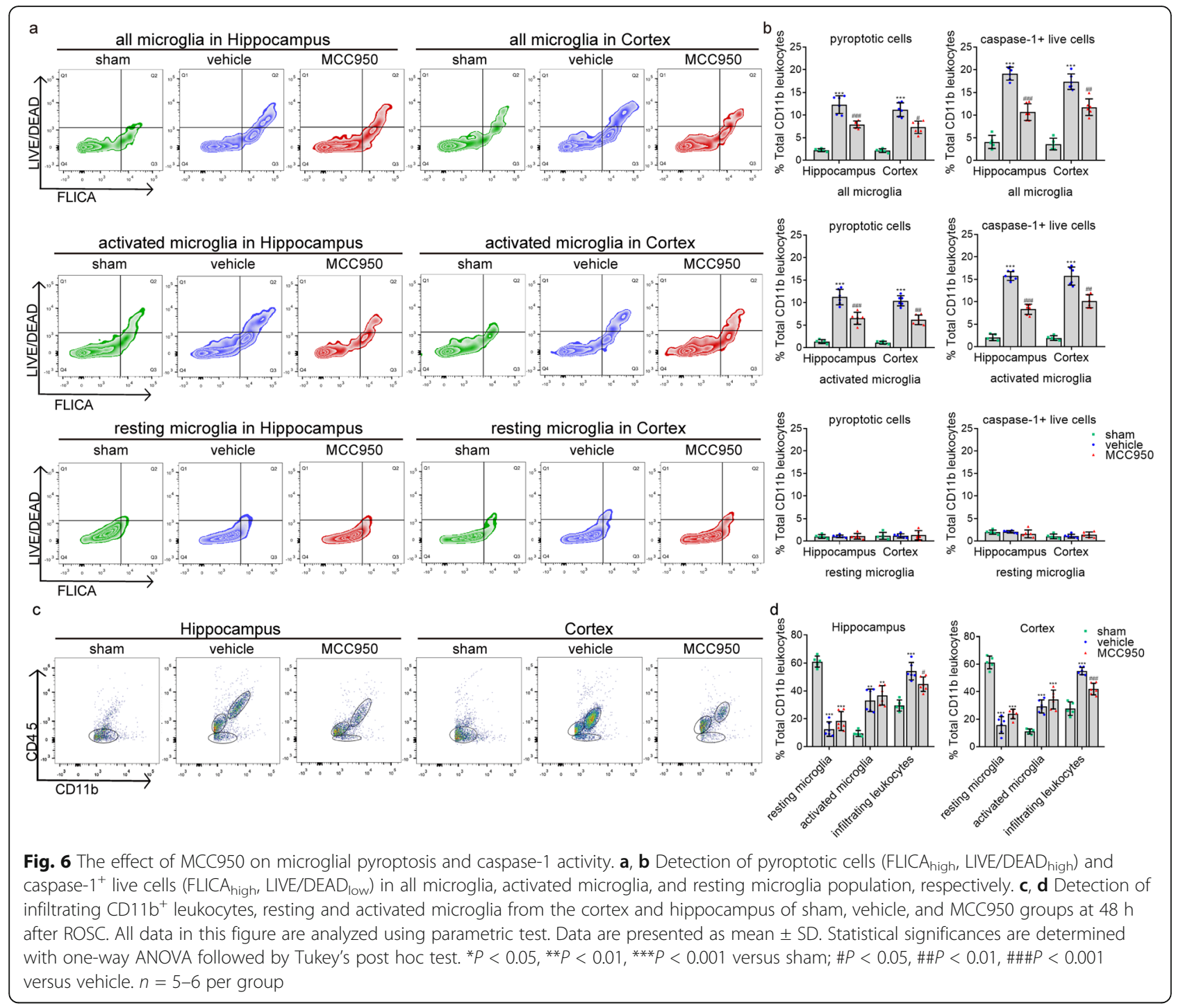

found that rats in the vehicle group presented lower NDSs at 24, 48, $72 \mathrm{~h}$ and on day 7 after ROSC than those in the MCC950 group (Fig. 7b, c), indicating that the neurologic deficit caused by cardiac arrest was alleviated by MCC 950 treatment.

We also performed the Morris water maze test to evaluate the effect of MCC950 on short-term spatial learning and memory ability after cardiac arrest. As a comparison, sham-operated rats were used in this part of the experiment. During the hidden-platform training on day 9 to day 12 after surgery, rats in the sham, vehicle, and MCC950 groups all showed a gradual decline in latency to find the hidden platform over time ( $F=$ 60.086, $P=0.000$ ) (Fig. $7 \mathrm{~d}$ ). In addition, analysis of the training data by repeated-measures ANOVA showed that escape latency differed significantly among the groups $(F=13.662, P=0.000)$, with no significant interaction between groups and time points $(F=2.455, P=$
0.113). Compared with sham operation, cardiac arrest led to extended latencies on day 10 to day 12 in the vehicle group (all $P<0.05$ vs. sham), whereas the extended latencies were partly prevented by MCC 950 treatment (all $P<0.05$ vs. vehicle) using the Tukey's post hoc test. The poor performance of vehicle animals to climb up the hidden platform should not be attributed to slower swimming speed because the mean swimming speed was comparable among the three groups (data not shown). On day 13 after cardiac arrest, all rats received the probe test to assess their short-term memory (Fig. 7e, f). Results showed that the frequency of crossing the platform area was lower in the vehicle group than the sham group, whereas this figure was increased after MCC 950 treatment. Besides, rats administrated with MCC950 spent markedly more time searching in the platform quadrant $(\mathrm{Q} 3)$ in comparison with vehicle rats, while vehicle-operated rats spent increased time in the other 


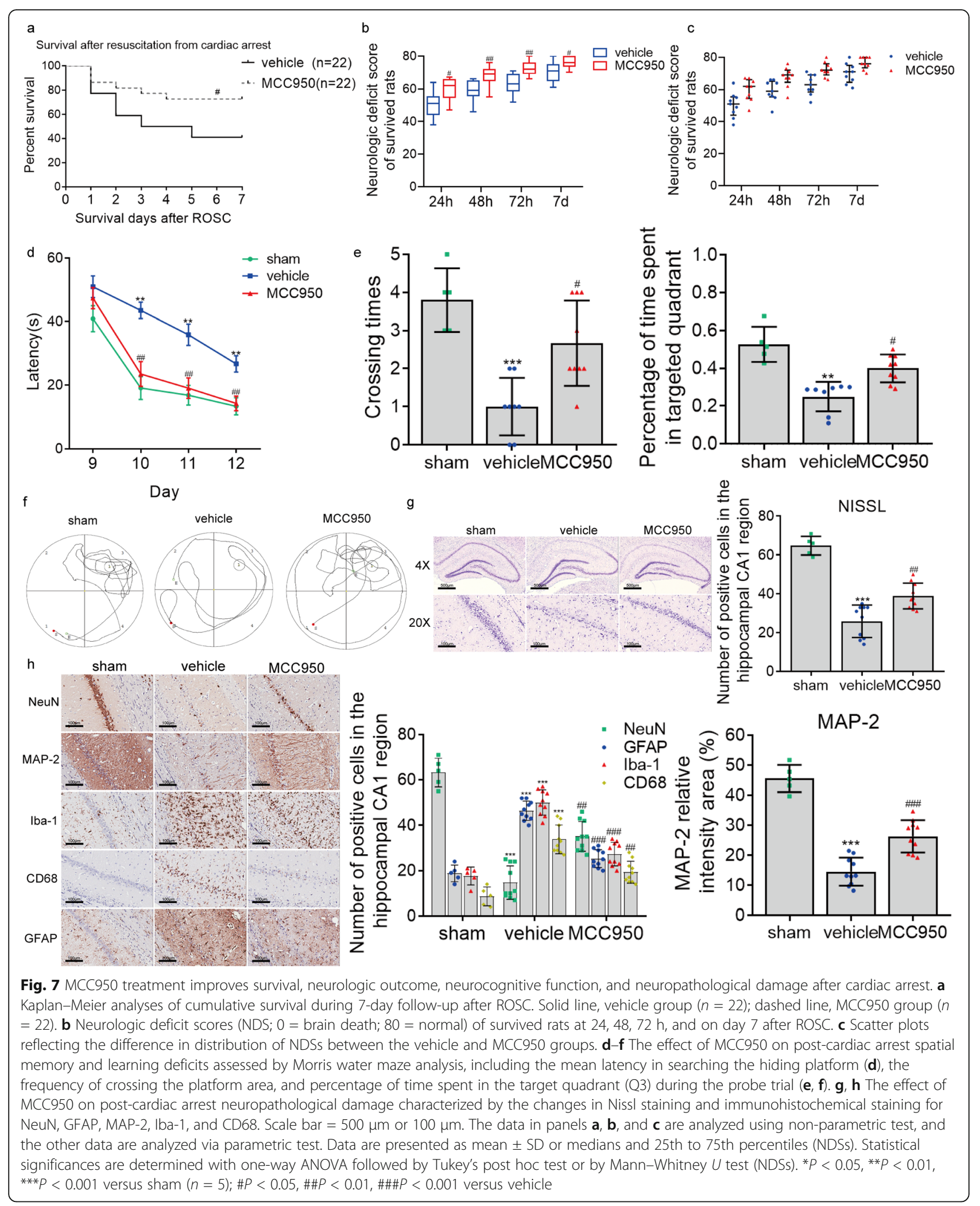


three non-platform quadrants (Fig. 7e, f). These findings from Morris water maze test indicate that MCC950 treatment rescued the spatial learning and memory deficiency caused by cardiac arrest.

These results demonstrate that long-term treatment with MCC950 after cardiac arrest provides persistent neuroprotection to improve 7-day survival and neurologic outcome.

\section{Targeting NLRP3 with MCC950 ameliorates histological injury after cardiac arrest}

To assess the histological damage caused by cardiac arrest and explore the profound effect of MCC950, all the rats involved in the Morris water maze test were euthanized on day 14 after cardiac arrest to conduct Nissl and immunohistochemical staining (Fig. 7g, h). The results from Nissl staining demonstrated that cardiac arrest induced neuron loss in the hippocampal CA1 region, a vulnerable region to global ischemia, whereas the neuron loss was partially restored by MCC950 (Fig. 7g). Consistently, less $\mathrm{NeuN}^{+}$cells (neuron) were noticed in the vehicle group compared to the sham group, while a significantly increased number of $\mathrm{NeuN}^{+}$cells was found in the MCC950 group. We also examined the injury to dendrite by immunostaining for MAP-2, a protein that was enriched in neuronal dendrites and acted as a stabilizing molecule for the dendritic cytoskeletal integrity (Fig. 7h). Results showed that cardiac arrest caused a dramatic dendritic loss in the vehicle group, as compared with sham controls. However, the dendritic loss was markedly reversed after MCC 950 treatment. These findings, therefore, indicate that MCC950 substantially prevents the neuron loss and dendritic injury induced by cardiac arrest.

Neuron loss is usually accompanied by glial activation to clean up the cell debris. As illustrated in Fig. 7h, microglia and astrocytes were dramatically activated in the post-cardiac arrest hippocampal CA1 region, as evidenced by increased immunoreactivities of Iba- 1 for microglia and GFAP for astrocytes. The number of Iba1-positive microglia and GFAP-positive astrocytes were both markedly reduced by MCC950 intervention compared to the vehicle group, indicating that the activation of microglia and astrocytes were inhibited by MCC 950 . Based on the results of flow cytometry, the number of activated microglia was elevated after cardiac arrest modeling, which was perceived as an important link to the pyroptosis implicated in the vigorous post-cardiac arrest inflammation and subsequent brain damage. We thus stained the brain sections with CD68 antibody, a marker for activated microglia, to further explore the inhibitory effect of MCC950 on the activated microglia. Unlike the findings observed at $48 \mathrm{~h}$ after cardiac arrest, there was a significant decrease in the intensity of CD68 staining in the presence of MCC950 treatment after cardiac arrest (Fig. 7h). This might be due to the inhibition of early inflammatory response by MCC 950 after cardiac arrest, thereby reducing the further activation of microglia. Taken together, these results suggest that MCC950 significantly prevents histological injuries, which might be via interfering NLRP3 inflammasome activation and suppressing microglia pyroptosis in the brain after cardiac arrest.

\section{Targeting caspase- 1 by Ac-YVAD-cmk prevents microglial pyroptosis and consequential neuroinflammation after cardiac arrest}

To further verify the role of NLRP3 inflammasome in mediating post-cardiac arrest microglial pyroptosis and its consequential brain injury, we used a selective inhibitor Ac-YVAD-cmk to target caspase-1, the canonical executor of pyroptosis [34]. In this part, rats were randomized to receive one dose of Ac-YVAD-cmk (400 ng in $4 \mu \mathrm{L}$, i.c.v.) or vehicle before the induction of asphyxial cardiac arrest (Fig. 1). There was no significant difference found in the ratio of achieving ROSC between the Ac-YVAD-cmk (35/ $42,83.3 \%)$ and vehicle $(35 / 44,79.5 \%)$ groups. Moreover, the body weight, the time from asphyxia to cardiac arrest, the time required for ROSC, epinephrine usage, and physiological variables were all comparable between these two groups (Table 2).

We found that the mRNA levels coding for IL- $1 \beta$ and IL-18 in hippocampus and cortex of post-cardiac arrest rats were downregulated after treatment with AcYVAD-cmk. However, there was no significant change for the mRNA levels of NLRP3, ASC, caspase-1, and GSDMD with Ac-YVAD-cmk treatment (Fig. 8a). AcYVAD-cmk treatment also significantly inhibited the activity of cleaved caspase- 1 and cleaved GSDMD, and decreased the protein level of pro-IL-1 $\beta$, pro-IL-18, IL$1 \beta$, and IL-18 in both hippocampus and cortex, while the levels of NLRP3 and ASC were not changed after Ac-YVAD-cmk treatment (Fig. 8b, c). These results were further confirmed by immunofluorescence staining. Inhibition of caspase- 1 by Ac-YVAD-cmk dramatically reduced cleaved caspase-1 but not NLRP3 or ASC, as compared with those vehicle controls (Fig. 8d, e). These findings suggest that Ac-YVAD-cmk restrains the cleavage of caspase- 1 , and thereby prevents the maturation of IL-1 $\beta$ and IL-18 in the brain after cardiac arrest.

We next detected whether the inhibition of caspase-1 by Ac-YVAD-cmk suppressed microglia pyroptosis and the caspase- 1 activity in activated microglia caused by cardiac arrest. As expected, targeting caspase- 1 by AcYVAD-cmk substantially reduced the number of pyroptotic cells and live cells expressing caspase- 1 in all microglia population $\left(\mathrm{CD} 45_{\text {low }} \mathrm{CD} 11 \mathrm{~b}^{+}\right.$and $\mathrm{CD} 45_{\text {int }}$ $\mathrm{CD}_{11 \mathrm{~b}^{+}}$) as well as in the $\mathrm{CD} 45_{\text {int }} \mathrm{CD} 11 \mathrm{~b}^{+}$population 
Table 2 Parameters of the of the Vehicle $\mathrm{A}_{\mathrm{A}}$ and Ac-YVAD-cmk groups at baseline and during post-cardiac arrest care after ROSC

\begin{tabular}{|c|c|c|c|}
\hline Parameters & Time points & Vehicle $_{\mathrm{A}}$ & Ac-YVAD-cmk \\
\hline Body weight, g & Baseline & $381 \pm 13$ & $374 \pm 17$ \\
\hline Time from asphyxia to cardiac arrest, $s$ & Baseline & $220.4 \pm 15$ & $217.1 \pm 23$ \\
\hline Time required for ROSC, s & Baseline & $99.06 \pm 17$ & $96.45 \pm 22$ \\
\hline Total dose of epinephrine, $\mu \mathrm{g}$ & Baseline & $12.3 \pm 3.1$ & $11.1 \pm 3.0$ \\
\hline \multirow[t]{4}{*}{ Mean arterial pressure, $\mathrm{mmHg}$} & Baseline & $119 \pm 7$ & $124 \pm 11$ \\
\hline & $10 \mathrm{~min}$ & $116 \pm 12$ & $107 \pm 7$ \\
\hline & $30 \mathrm{~min}$ & $84 \pm 9$ & $83 \pm 5$ \\
\hline & $60 \mathrm{~min}$ & $80 \pm 5$ & $81 \pm 11$ \\
\hline \multirow[t]{4}{*}{ Heart rate, beats/min } & Baseline & $407 \pm 11$ & $403 \pm 7$ \\
\hline & $10 \mathrm{~min}$ & $431 \pm 15$ & $427 \pm 19$ \\
\hline & $30 \mathrm{~min}$ & $372 \pm 11$ & $369 \pm 17$ \\
\hline & $60 \mathrm{~min}$ & $383 \pm 17$ & $379 \pm 15$ \\
\hline \multirow[t]{4}{*}{ Rectal temperature, ${ }^{\circ} \mathrm{C}$} & Baseline & $36.8 \pm 0.4$ & $36.9 \pm 0.2$ \\
\hline & $10 \mathrm{~min}$ & $37.1 \pm 0.3$ & $36.9 \pm 0.3$ \\
\hline & $30 \mathrm{~min}$ & $37.0 \pm 0.2$ & $36.8 \pm 0.3$ \\
\hline & $60 \mathrm{~min}$ & $36.8 \pm 0.2$ & $36.9 \pm 0.2$ \\
\hline
\end{tabular}

after cardiac arrest (Fig. 9a, b). Again, the suppression of microglial pyroptosis by Ac-YVAD-cmk led to a notably decreased number of $\mathrm{CD} 45_{\text {high }} \mathrm{CD} 11 \mathrm{~b}^{+}$population compared to the vehicle group (Fig. 9c, d). Thus, targeting caspase-1 by Ac-YVAD-cmk prevents microglial pyroptosis and consequential neuroinflammation after cardiac arrest.

\section{Targeting caspase- 1 by Ac-YVAD-cmk ameliorates neurological injury after cardiac arrest}

We then assessed whether the suppression of microglia pyroptosis by targeting caspase-1 with Ac-YVAD-cmk ameliorated neurological injury in a rat model of cardiac arrest. Post-cardiac arrest rats in the Ac-YVAD-cmk group exhibited statistically higher NDSs at $24,48,72 \mathrm{~h}$, and 7 days than those in the vehicle group (Fig. 10a, b), suggesting that Ac-YVAD-cmk intervention reduced neurologic damage. Furthermore, during the training period of the Morris water maze test, the escape latency became progressively shorter in all groups over time $(F$ $=71.673, P=0.000$ ), and repeated-measures ANOVA revealed that escape latency differed significantly among the groups $(F=13.768, P=0.000)$, with no significant interaction between groups and time points $(F=2.040$, $P=0.075)$. Besides, Tukey's post hoc test showed that Ac-YVAD-cmk substantially shorted the latency in finding the hidden platform on day 10,11, and 12 after cardiac arrest modeling groups (Fig. 10c), while the mean swimming speed was comparable between the AcYVAD-cmk and the vehicle groups (data not shown). In the probe trial of the Morris water maze test, the frequency of crossing the platform area was increased in the Ac-YVAD-cmk-treated group compared with the vehicle-treated groups (Fig. 10d, e). Moreover, rats with an intracerebroventricular injection of Ac-YVAD-cmk had a trend toward spending more time searching in the platform quadrant $(\mathrm{Q} 3)$ in comparison with vehicle rats, while vehicle-operated rats seemed to spend increased time in the other three non-platform quadrants (Fig. 10d, e).

After the Morris water maze test, all rats in this part of the experiment were euthanized, and brain sections were prepared for histological injury evaluation. Our results of Nissl staining revealed that there were significantly more viable neurons in the Ac-YVAD-cmktreated group compared to the vehicle group (Fig. 10f). Ac-YVAD-cmk-treated rats exhibited more $\mathrm{NeuN}^{+}$neurons in hippocampal CA1 region than vehicle-treated rats (Fig. 10g). Besides, post-cardiac arrest rats in the vehicle group exhibited extensive loss of MAP2immunoreactive dendrites in the hippocampus CA1 region, which was partially recovered by Ac-YVAD-cmk treatment (Fig. 10g). We next carried out the Iba-1, CD68, and GFAP immunohistochemical staining to verify the activation of microglia and astrocytes. As expected, extensive Iba-1-positive, GFAP-positive, and CD68-positive cells appeared in the hippocampal CA1 region in the post-cardiac arrest rats treated with vehicle solution compared to the sham controls. However, AcYVAD-cmk treatment significantly reduced Iba-1positive, GFAP-positive, and CD68-positive cells in the hippocampal CA1 region of post-cardiac arrest rats. 


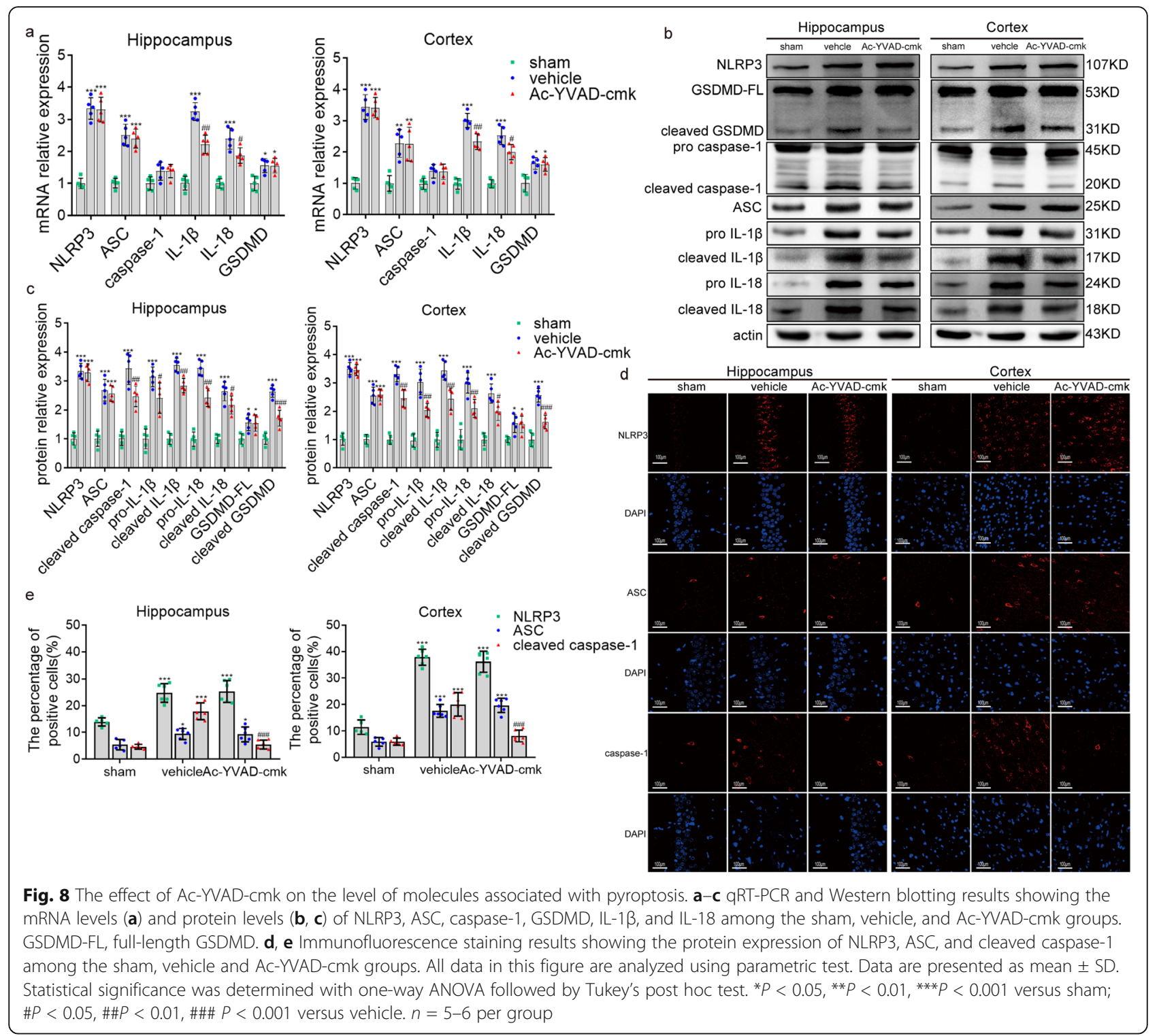

These results suggest that pharmacological blockade of caspase- 1 by Ac-YVAD-cmk considerably suppressed cardiac arrest-induced activation of microglia and astrocytes. Overall, the findings from this part suggest that pre-treatment with Ac-YVAD-cmk provides profound effects in preventing neurologic deficit and alleviating neuropathological injury induced by cardiac arrest, which could be attributed to the suppression of microglial pyroptosis and ensuing inflammatory responses.

\section{Discussion}

Asphyxia caused by foreign-body airway obstruction, hanging, or strangulation is a common pathogenesis of cardiac arrest [35]. In this study, we used a 10-min asphyxial cardiac arrest and cardiopulmonary resuscitation model to reproduce the pathophysiology of hypoxic- ischemic encephalopathy and histological injury similar to asphyxial humans [36, 37]. We demonstrate that (1) cardiac arrest leads to microglial activation and leukocytes infiltration in the brain; (2) cardiac arrest triggers microglial pyroptosis and increases the caspase-1 activity in the activated microglia population, which is mediated by the NLRP3 inflammasome; and (3) targeting NLRP3 and caspase-1 with MCC950 and AcYVAD-cmk significantly prevent microglial pyroptosis and consequential inflammatory response after cardiac arrest, which further leads to improved neurologic outcome and less histological injury in cardiac arrestmodeling rats. Our results, for the first time, highlight the significance of NLRP3 inflammasome-mediated microglial pyroptosis in the development of postcardiac arrest brain injury. 


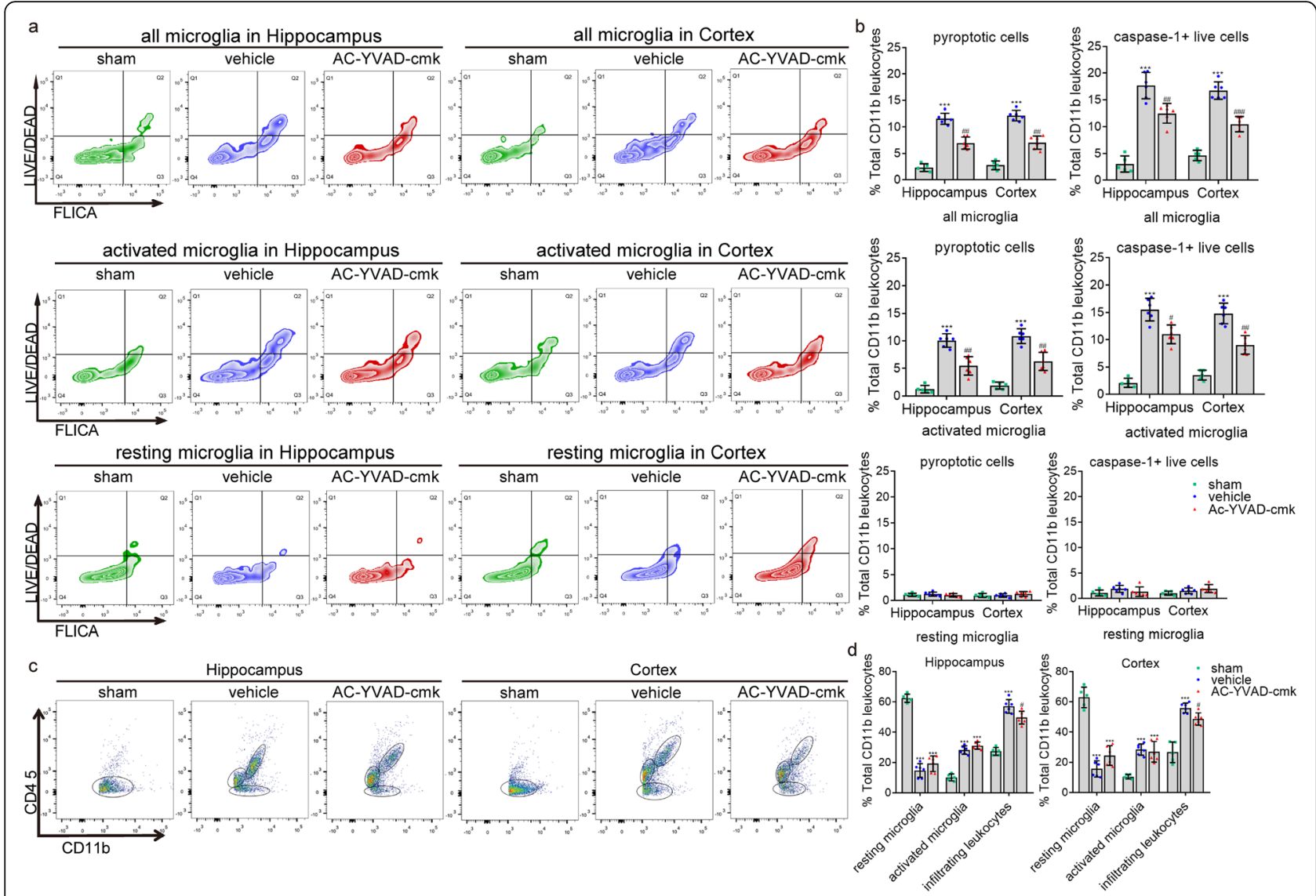

Fig. 9 The effect of Ac-YVAD-cmk on microglial pyroptosis and caspase-1 activity. $\mathbf{a}$, b Detection of pyroptotic cells (FLICA $A_{\text {high, }}$ LIVE/DEAD high) and caspase- $1^{+}$live cells (FLICA high, $_{\text {LIVE/DEAD }}$ low) in all microglia, activated microglia, and resting microglia population, respectively. $\mathbf{c}$, $\mathbf{d}$ Detection of infiltrating CD1 1 b leukocytes, resting, and activated microglia from the cortex and hippocampus of sham, vehicle, and Ac-YVAD-cmk groups. All data in this figure are analyzed using parametric test. Data are presented as mean \pm SD. Statistical significance was determined with one-way ANOVA

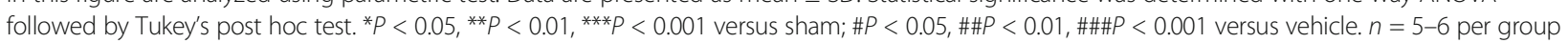

Sterile inflammation has been increasingly recognized as an important factor leading to secondary brain injury in a variety of neuropathic conditions, where the activation of microglia, the resident immune cells in the brain, is the initial step in the inflammatory responses [38]. This initial step is followed by the infiltration of circulating monocytes, neutrophils and $\mathrm{T}$ cells [39], which further amplifies the inflammatory response in a stimulated brain. Consistent with these theories, in this study, we demonstrate that microglia were extensively activated in the post-cardiac arrest brain, accompanying exudation of vast myeloid-lineage leukocytes to the brain parenchyma, which suggests that microglial activation and ensuing inflammatory response indeed are critically involved in the pathogenesis of post-cardiac arrest brain injury.

Recent studies have reported that pyroptosis, a ubiquitous form of proinflammatory programmed cell death causing a cascade of inflammatory responses, is implicated in various neurological stimuli [40]. More recently, microglial pyroptosis caused by inflammasome activation and infiltrating leukocytes is shown to be critically involved in the pathophysiology of penetrating traumatic brain injury [13]. Canonical pyroptosis is executed by the cleaved caspase-1, which not only causes cell lysis during pyroptosis but also mediates proteolytic cleavage and release of IL-1 $\beta$ and IL-18 [41]. These IL-1 $\beta$ and IL18 , in turn, propagate the neuroinflammatory response through further activation of resident cells (i.e., microglia) and recruitment of inflammatory cells, such as neutrophils, macrophages, and lymphocytes [40]. Given the over-activation of microglia and the increment in inflammatory cytokines after cardiac arrest as found in this and our previous studies [15, 20,42-44], we proposed that the overactivated microglia may undergo pyroptosis after cardiac arrest and aggravates the subsequent inflammation in the brain. Consistent with this hypothesis, our data demonstrate that there is indeed intensive pyroptosis and increased caspase- 1 activity in the activated microglia population after cardiac arrest, along with 


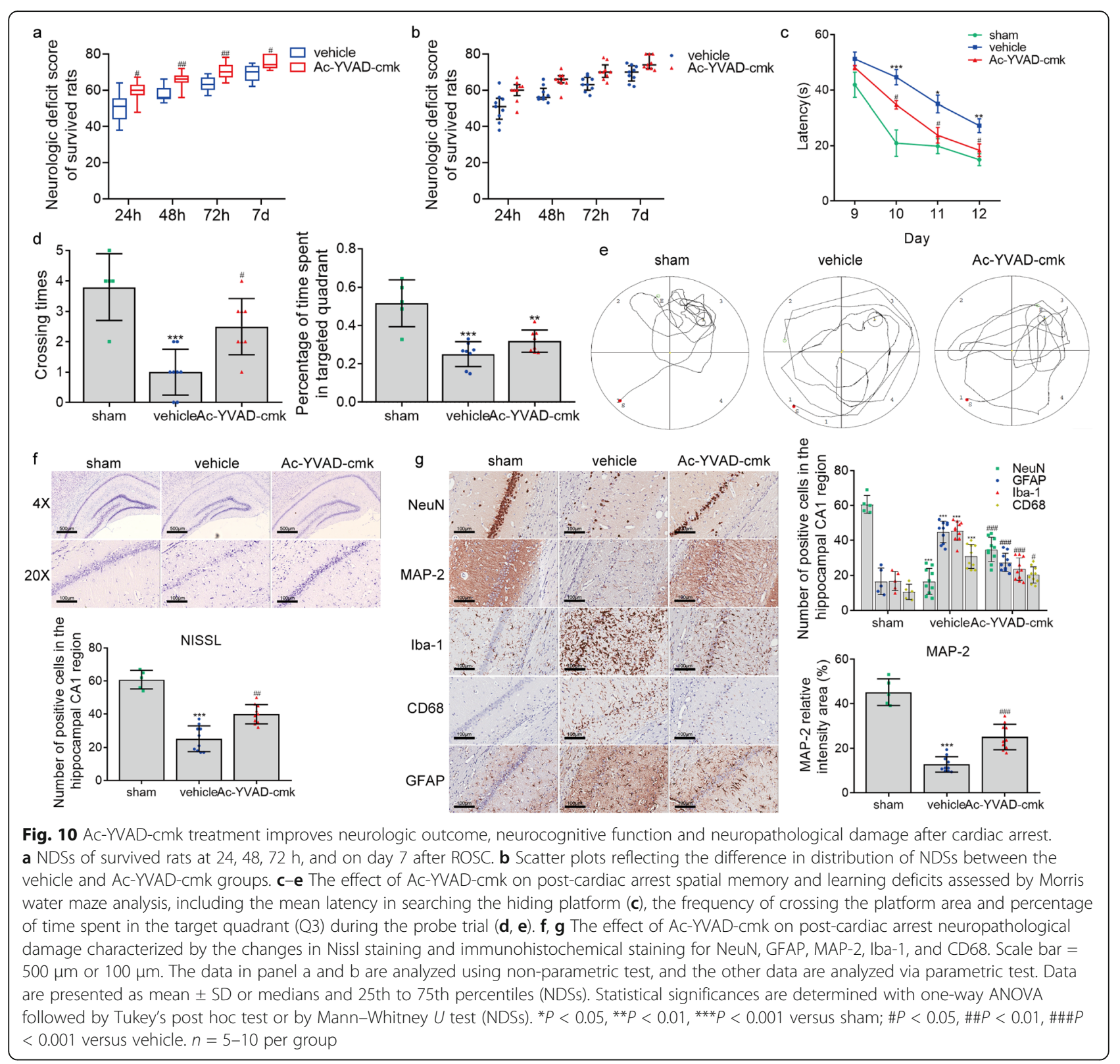

elevated levels of IL-1 $\beta$ and IL-18. Moreover, blockage of caspase- 1 by a selective inhibitor, Ac-YVAD-cmk, suppresses the pyroptosis, IL-1 $\beta$ and IL-18 levels, as well as inflammatory infiltration but not the number of activated microglia, indicating that the activation of microglia occurs before pyroptosis. Therefore, we provide evidence that microglia are conversed from resting state into activated state after cardiac arrest, followed by the occurrence of pyroptosis and consequential aggravation of sterile inflammation in the brain.

The cleaved caspase- 1 comes from autocatalysis and activation of pro-caspase-1 under a platform provided by the assembly of inflammasomes, which are cytosolic protein complexes that contain different structural domains that mediate individual functions [45]. In microglia, the best characterized component is the NLRP3 inflammasome comprised of NLRP3, the adaptor ASC, and pro-caspase-1 [45]. Similar to other NLRs, NLRP3 contains an N-terminal effector binding domain, a nucleotide-binding oligomerization domain, and a $\mathrm{C}$-terminal leucine-rich repeat receptor domain, which bind to ligands and lead to activation of inflammasomes [46]. Most inflammasomes also have ASC as an adaptor molecule, which translocates to the cytoplasm in response to stimuli to form specks and recruit caspase-1 [45]. Under pathological conditions such as amyotrophic lateral sclerosis [47], danger-associated molecular patterns produced by 
host cells [e.g., ATP, DNA, reactive oxygen species] can initiate the activation of inflammasome, presenting as elevated level and interaction of the components of the inflammasome. Here, we revealed that NLRP3 and ASC were upregulated in the post-cardiac arrest brain with biological interaction, and both NLRP3 and ASC had interaction with caspase-1, which represent the intracellular activation of NLRP3 inflammasome [27]. Besides, NLRP3 and caspase-1 were mainly co-localized in microglia but not neuron, astrocytes, endothelial cells, or oligodendrocyte. Moreover, targeting NLRP3 by MCC950 suppresses the cleavage of caspase-1, elevated levels of IL-1 $\beta$ and IL18 , microglial pyroptosis, as well as infiltration of leucocytes. Therefore, for the first time, we show that the NLRP3 inflammasome-mediated microglial pyroptosis is crucially involved in the development of neuroinflammation after cardiac arrest.

In addition to regulating neuroinflammation, we are more interested in whether the intervention in NLRP3 inflammasome translates into improvements in neurologic outcome and tissue damage. Therefore, we conducted randomized, vehicle-controlled animal studies with blind outcome evaluation to assess the role of MCC950 and Ac-YVAD-cmk in post-cardiac arrest brain injury. MCC950 is a novel, selective smallmolecule inhibitor that specifically blocks NLRP3 activation [48], whereby it attenuates inflammation and brain injury after intracerebral hemorrhage and ischemic stroke $[49,50]$. Consistent with these findings, we demonstrated that MCC950 showed benefit in improving survival, neurologic function, and lessening neuropathological injuries in post-cardiac arrest rats, indicating that MCC950 may target the NLRP3 inflammasome to prevent post-cardiac arrest brain damage. Ac-YVAD-cmk is an irreversible tetrapeptide inhibitor of caspase-1 with good cell permeability and protects the brain against injury induced by the stimuli such as intracerebral hemorrhage [51]. Here, we add to the current knowledge that the delivery of Ac-YVAD-cmk before cardiac arrest affords neuroprotection against brain injury. It is worth noting that in this study, the two drugs have different effects on improving short-term memory and cognitive function of rats after cardiac arrest, which is reflected in the result that there was no significant difference in the percentage of the time in the target quadrant on probe test after Ac-YVAD-cmk pre-treatment, compared to the vehicle group. In our opinion, the possible reasons for this difference are as follows; firstly, the different routes of administration of the two drugs might have influenced the onset time and efficacy of the drugs. Secondly, we determined the concentrations and doses of the two drugs based on previous studies, which might not be equivalent. To sum up, targeting NLRP3 inflammasome suppresses microglial pyroptosis and neuroinflammation, which further leads to prevention of post-cardiac arrest brain injury.

In this study, we delivered the systemic damage in rats using cardiac arrest modeling, possibly injuring organs other than brain including liver and kidney, whose dysfunction can trigger or aggravate encephalopathy. However, no significant impairment of liver and kidney function was observed in the modeling rats used in our study, and our unpublished data showed no significant changes in creatinine, alanine aminotransferase, glutamate aminotransferase and blood ammonia levels of the post-cardiac arrest animals. Of course, we cannot eliminate that the activation of NLRP3 inflammasome exists in the liver or kidney after cardiac arrest at the molecular level, and pharmacologic blockage of NLRP3 inflammasome may exert a protective effect on these tissues. This should be a limitation of this study.

In summary, we provide evidence that cardiac arrest induces the assembly of NLRP3 inflammasome, which triggers microglial pyroptosis and consequential neuroinflammation and ultimately aggravates brain injury. This work opens up possibilities for further studies to better understand the pathogenesis of post-cardiac arrest brain damage. Furthermore, the demonstration that MCC950 and Ac-YVAD-cmk confer neuroprotection in the model of cardiac arrest advances the current therapies and highlights an under-appreciated effect of these experimental drugs. Although it still requires time to be switched into clinical medication, MCC950 and Ac-YVAD-cmk represent novel and significant strategies to target or address post-cardiac arrest brain injury.

\section{Conclusion}

This study illuminates that microglial pyroptosis mediated by NLRP3 inflammasome is closely related to the severe neuroinflammation after cardiac arrest and critically involved in the pathogenesis of post-cardiac arrest brain injury. In addition, our results provide evidence that MCC950 and Ac-YVAD-cmk could ameliorate neurologic injuries thus helping better outcome, and MCC950 could also improve survival after cardiac arrest, possibly via preventing the activation of NLRP3 inflammasome, the occurrence of microglial pyroptosis, and consequential inflammatory response. Hence, this study provides a new therapeutic strategy.

\section{Abbreviations}

NLRP3: Nod-like receptor family protein 3; IL-1B: Interleukin 1B; IL18: Interleukin 18; pro-IL-1 $\beta$ : Precursors of IL-1 $\beta$; pro-IL-18: Precursors of IL-18; ASC: Apoptosis-associated speck-like proteins containing a caspase recruitment domain; pro-caspase-1: Precursor of caspase-1; GSDMD: Gasdermin D; GSDMD-FL: Full-length GSDMD; pro-caspase-11: Precursor of caspase-11; DMSO: Dimethyl sulfoxide; NDS: Neurologic deficit score; NeuN: Neuronal nuclei; MAP2: Microtubule-associated protein 2; GFAP: Glial fibrillary acidic 
protein; Iba-1: Ionized calcium-binding adapter molecule-1; Olig2: Oligodendrocyte lineage transcription factor 2; ROSC: Return of spontaneous circulation; ECG: Electrocardiogram; MAP: Mean arterial pressure; HR: Heart rate; CA/CPR: Cardiac arrest and cardiopulmonary resuscitation; ACA: Asphyxial cardiac arrest; qRT-PCR: Quantitative real-time polymerase chain reaction

\section{Acknowledgements}

We thank Bibo Ke, from the Dumont-UCLA Transplant Center, Department of Surgery, David Geffen School of Medicine at UCLA, for helpful suggestions on the study design and manuscript preparation.

\section{Authors' contributions}

YC, JZ, DW, HL, YH, KL, XW, YP, SP, and KH performed all the experiments. $\mathrm{YC}, \mathrm{SP}$, and $\mathrm{KH}$ performed data analysis and wrote the manuscript. All authors read and approved the final manuscript.

\section{Funding}

This study was supported by the National Natural Science Foundation of China (Grant No. 81701294 \& 81871030), Guangzhou Science and Technology Planning Project (Grant No. 201804010055), the National Key R\&D Program of China (Grant No. 2017YFC1307501), and Outstanding Youths Development Scheme of Nanfang Hospital, Southern Medical University (2016J005).

\section{Availability of data and materials}

The datasets used and/or analyzed during this study are available from the corresponding authors on reasonable request.

\section{Ethics approval}

All animal experiments in this study were approved by Animal Care and Use Committee of Nanfang Hospital, Southern Medical University (Guangzhou, China).

\section{Consent for publication}

$$
\text { Not applicable. }
$$

\section{Competing interests}

The authors declare that they have no competing interests.

\section{Author details}

'Department of Neurology, Nanfang Hospital, Southern Medical Univerisity, North Avenue 1838\#, Guangzhou, Guangzhou 510515, China. ²Department of Dermatology, Zhujiang Hospital, Southern Medical University, Guangzhou, Guangdong, China. ${ }^{3}$ Department of Neurology, Zhuhai Hospital of Integrated Traditional Chinese and Western Medicine, Zhuhai, China.

\section{Received: 10 January 2020 Accepted: 23 June 2020}

\section{Published online: 23 July 2020}

\section{References}

1. Shao F, Li CS, Liang LR, Li D, Ma SK. Outcome of out-of-hospital cardiac arrests in Beijing China. Resuscitation. 2014;85:1411-7.

2. Mozaffarian D, Benjamin EJ, Go AS, Arnett DK, Blaha MJ, Cushman M, Das SR, de Ferranti S, Despres JP, Fullerton HJ, et al. Heart disease and stroke statistics-2016 update: a report from the American Heart Association. Circulation. 2016;133:e38-360

3. Neumar RW, Nolan JP, Adrie C, Aibiki M, Berg RA, Bottiger BW, Callaway C, Clark RS, Geocadin RG, Jauch EC, et al. Post-cardiac arrest syndrome: epidemiology, pathophysiology, treatment, and prognostication. A consensus statement from the International Liaison Committee on Resuscitation (American Heart Association, Australian and New Zealand Council on Resuscitation, European Resuscitation Council, Heart and Stroke Foundation of Canada, InterAmerican Heart Foundation, Resuscitation Council of Asia, and the Resuscitation Council of Southern Africa); the American Heart Association Emergency Cardiovascular Care Committee; the Council on Cardiovascular Surgery and Anesthesia; the Council on Cardiopulmonary, Perioperative, and Critical Care; the Council on Clinical Cardiology; and the Stroke Council. Circulation. 2008;118:2452-83.

4. Liu F, McCullough LD. Inflammatory responses in hypoxic ischemic encephalopathy. Acta Pharmacol Sin. 2013;34:1121-30.
5. Fink SL, Cookson BT. Apoptosis, pyroptosis, and necrosis: mechanistic description of dead and dying eukaryotic cells. Infect Immun. 2005;73: 1907-16.

6. Man SM, Karki R, Kanneganti TD. Molecular mechanisms and functions of pyroptosis, inflammatory caspases and inflammasomes in infectious diseases. Immunol Rev. 2017;277:61-75.

7. Shi J, Zhao Y, Wang K, Shi X, Wang Y, Huang H, Zhuang Y, Cai T, Wang F, Shao F. Cleavage of GSDMD by inflammatory caspases determines pyroptotic cell death. Nature. 2015;526:660-5.

8. de Rivero VJ, Dietrich WD, Keane RW. Activation and regulation of cellular inflammasomes: gaps in our knowledge for central nervous system injury. J Cereb Blood Flow Metab. 2014;34:369-75.

9. Trendelenburg G. Molecular regulation of cell fate in cerebral ischemia: role of the inflammasome and connected pathways. J Cereb Blood Flow Metab. 2014;34:1857-67.

10. Xu P, Zhang X, Liu Q, Xie Y, Shi X, Chen J, Li Y, Guo H, Sun R, Hong Y, et al. Microglial TREM-1 receptor mediates neuroinflammatory injury via interaction with SYK in experimental ischemic stroke. Cell Death Dis. 2019; 10:555.

11. Zhao $H$, Chen $Y$, Feng $H . P 2 X 7$ receptor-associated programmed cell death in the pathophysiology of hemorrhagic stroke. Curr Neuropharmacol. 2018; 16:1282-95.

12. Bobinger $T$, Burkardt $P$, Huttner HB, Manaenko A. Programmed cell death after intracerebral hemorrhage. Curr Neuropharmacol. 2018;16:1267-81.

13. Lee SW, de Rivero VJ, Truettner JS, Dietrich WD, Keane RW. The role of microglial inflammasome activation in pyroptotic cell death following penetrating traumatic brain injury. J Neuroinflammation. 2019;16:27.

14. Ward R, Li W, Abdul Y, Jackson L, Dong G, Jamil S, Filosa J, Fagan SC, Ergul A. NLRP3 inflammasome inhibition with MCC950 improves diabetesmediated cognitive impairment and vasoneuronal remodeling after ischemia. Pharmacol Res. 2019;142:237-50.

15. Huang KB, Wang ZY, Gu Y, Hu YF, Ji Z, Wang SN, Lin ZZ, Li X, Xie ZS, Pan SY. Glibenclamide is comparable to target temperature management in improving survival and neurological outcome after asphyxial cardiac arrest in rats. J Am Heart Assoc. 2016;5:e3456.

16. Coll RC, Robertson AA, Chae JJ, Higgins SC, Munoz-Planillo R, Inserra MC, Vetter I, Dungan LS, Monks BG, Stutz A. A small-molecule inhibitor of the NLRP3 inflammasome for the treatment of inflammatory diseases. Nat Med. 2015;21:248-55.

17. Ren H, Kong Y, Liu Z, Zang D, Yang X, Wood K, Li M, Liu Q. Selective NLRP3 (pyrin domain-containing protein 3) inflammasome inhibitor reduces brain injury after intracerebral hemorrhage. Stroke. 2018;49:184-92.

18. Qi Y, Klyubin I, Cuello AC, Rowan MJ. NLRP3-dependent synaptic plasticity deficit in an Alzheimer's disease amyloidosis model in vivo. Neurobiol Dis. 2018:114:24-30

19. Rabuffetti M, Sciorati C, Tarozzo G, Clementi E, Manfredi AA, Beltramo M. Inhibition of caspase-1-like activity by Ac-Tyr-Val-Ala-Asp-chloromethyl ketone induces longlasting neuroprotection in cerebral ischemia through apoptosis reduction and decrease of proinflammatory cytokines. J Neurosci. 2000;20:4398-404.

20. Huang KB, Gu Y, Hu YF, Ji Z, Wang SN, Lin ZZ, Li X, Xie ZS, Pan SY. Glibenclamide improves survival and neurologic outcome after cardiac arrest in rats. Crit Care Med. 2015;43:e341-9.

21. Geocadin RG, Ghodadra R, Kimura T, Lei H, Sherman DL, Hanley DF, Thakor NV. A novel quantitative EEG injury measure of global cerebral ischemia. Clin Neurophysiol. 2000;111:1779-87.

22. Hickey RW, Akino M, Strausbaugh S, De Courten-Myers GM. Use of the Morris water maze and acoustic startle chamber to evaluate neurologic injury after asphyxial arrest in rats. Pediatr Res. 1996;39:77-84.

23. Truettner JS, Bramlett HM, Dietrich WD. Hyperthermia and mild traumatic brain injury: effects on inflammation and the cerebral vasculature. J Neurotraum. 2018;35:940-52.

24. Truettner JS, Bramlett HM, Dietrich WD. Posttraumatic therapeutic hypothermia alters microglial and macrophage polarization toward a beneficial phenotype. J Cereb Blood Flow Metab. 2017;37:2952-62.

25. Greter M, Lelios I, Croxford AL. Microglia versus myeloid cell nomenclature during brain inflammation. Front Immunol. 2015:6:249.

26. Ford AL, Goodsall AL, Hickey WF, Sedgwick JD. Normal adult ramified microglia separated from other central nervous system macrophages by flow cytometric sorting. Phenotypic differences defined and direct ex vivo antigen presentation to myelin basic protein- reactive CD4+ T cells compared. J Immunol. 1995;154:4309-21. 
27. Walsh JG, Muruve DA, Power C. Inflammasomes in the CNS. Nat Rev Neurosci. 2014;15:84-97.

28. Xi H, Zhang $Y, X u$ Y, Yang WY, Jiang $X$, Sha $X$, Cheng $X$, Wang J, Qin $X$, Yu J, et al. Caspase-1 inflammasome activation mediates homocysteine-induced pyrop-apoptosis in endothelial cells. Circ Res. 2016;118:1525-39.

29. Rathinam VAK, Zhao Y, Shao F. Innate immunity to intracellular LPS. Nat Immunol. 2019;20:527-33.

30. Wang Z, Meng S, Cao L, Chen Y, Zuo Z, Peng S. Critical role of NLRP3caspase-1 pathway in age-dependent isoflurane-induced microglial inflammatory response and cognitive impairment. J Neuroinflamm. 2018;15: 109-12.

31. Cheon SY, Kim JM, Kim EJ, Kim SY, Kam EH, Ho CC, Lee SK, Koo BN. Intranuclear delivery of synthetic nuclear factor-kappa B p65 reduces inflammasomes after surgery. Biochem Pharmacol. 2018;158:141-52.

32. Wan F, Lenardo MJ. The nuclear signaling of NF-KB: current knowledge, new insights, and future perspectives. Cell Res. 2010;20:24-33.

33. Yamamoto Y, Gaynor RB. Therapeutic potential of inhibition of the NF-kB pathway in the treatment of inflammation and cancer. J Clin Invest. 2001; 107:135-42.

34. Miao EA, Rajan JV, Aderem A. Caspase-1-induced pyroptotic cell death. Immunol Rev. 2011;243:206-14.

35. Kitamura T, Kiyohara K, Sakai T, Iwami T, Nishiyama C, Kajino K, Nishiuchi T, Hayashi Y, Katayama Y, Yoshiya K, et al. Epidemiology and outcome of adult out-of-hospital cardiac arrest of non-cardiac origin in Osaka: a populationbased study. Bmj Open. 2014;4:e6462.

36. Xiao F, Pardue S, Arnold T, Carden D, Alexander JS, Monroe J, Sharp CD, Turnage $\mathrm{R}$, Conrad $\mathrm{S}$. Effect of ifenprodil, a polyamine site NMDA receptor antagonist, on brain edema formation following asphyxial cardiac arrest in rats. Resuscitation. 2004;61:209-19.

37. Katz LM, Young A, Frank JE, Wang Y, Park K. Neurotensin-induced hypothermia improves neurologic outcome after hypoxic-ischemia. Crit Care Med. 2004:32:806-10.

38. Shi K, Tian DC, Li ZG, Ducruet AF, Lawton MT, Shi FD. Global brain inflammation in stroke. Lancet Neurol. 2019:18:1058-66.

39. Zheng Z, Yenari MA. Post-ischemic inflammation: molecular mechanisms and therapeutic implications. Neurol Res. 2004:26:884-92.

40. Barrington J, Lemarchand E, Allan SM. A brain in flame; do inflammasomes and pyroptosis influence stroke pathology? Brain Pathol. 2017;27:205-12.

41. Bergsbaken T, Fink SL, Cookson BT. Pyroptosis: host cell death and inflammation. Nat Rev Microbiol. 2009:7:99-109.

42. Shi X, Li M, Huang K, Zhou S, Hu Y, Pan S, Gu Y. HMGB1 binding heptamer peptide improves survival and ameliorates brain injury in rats after cardiac arrest and cardiopulmonary resuscitation. Neuroscience. 2017;360:128-38.

43. Zhu J, Liu KW, Huang KB, Gu Y, Hu YF, Pan SY, Ji Z. Metformin improves neurologic outcome via AMP-activated protein kinase-mediated autophagy activation in a rat model of cardiac arrest and resuscitation. J Am Heart Assoc. 2018;7:e8389.

44. Huang KB, Wang ZY, Gu Y, Ji Z, Lin ZZ, Wang SN, Pan SY, Wu YM Glibenclamide prevents water diffusion abnormality in the brain after cardiac arrest in rats. Neurocrit Care. 2018;29:128-35.

45. Mamik MK, Power C. Inflammasomes in neurological diseases: emerging pathogenic and therapeutic concepts. Brain. 2017;140:2273-85.

46. Kita T, Miyazaki I, Asanuma M, Takeshima M, Wagner GC. Dopamineinduced behavioral changes and oxidative stress in methamphetamineinduced neurotoxicity. Int Rev Neurobiol. 2009;88:43-64.

47. Johann S, Heitzer M, Kanagaratnam M, Goswami A, Rizo T, Weis J, Troost D, Beyer C. NLRP3 inflammasome is expressed by astrocytes in the SOD1 mouse model of ALS and in human sporadic ALS patients. Glia. 2015;63: 2260-73.

48. van Hout GP, Bosch L, Ellenbroek GH, de Haan JJ, van Solinge WW, Cooper MA, Arslan F, de Jager SC, Robertson AA, Pasterkamp G, et al. The selective NLRP3-inflammasome inhibitor MCC950 reduces infarct size and preserves cardiac function in a pig model of myocardial infarction. Eur Heart J. 2017; 38:828-36.

49. Valderrama JA, Riestra AM, Gao NJ, LaRock CN, Gupta N, Ali SR, Hoffman HM, Ghosh P, Nizet V. Group A streptococcal M protein activates the NLRP3 inflammasome. Nat Microbiol. 2017;2:1425-34.

50. Ye X, Shen T, Hu J, Zhang L, Zhang Y, Bao L, Cui C, Jin G, Zan K, Zhang Z, et al. Purinergic $2 X 7$ receptor/NLRP3 pathway triggers neuronal apoptosis after ischemic stroke in the mouse. Exp Neurol. 2017;292:46-55.
51. Wu B, Ma Q, Khatibi N, Chen W, Sozen T, Cheng O, Tang J. Ac-YVAD-CMK decreases blood-brain barrier degradation by inhibiting caspase-1 activation of interleukin-1 beta in intracerebral hemorrhage mouse model. Transl Stroke Res. 2010:1:57-64.

\section{Publisher's Note}

Springer Nature remains neutral with regard to jurisdictional claims in published maps and institutional affiliations.
Ready to submit your research? Choose BMC and benefit from:

- fast, convenient online submission

- thorough peer review by experienced researchers in your field

- rapid publication on acceptance

- support for research data, including large and complex data types

- gold Open Access which fosters wider collaboration and increased citations

- maximum visibility for your research: over $100 \mathrm{M}$ website views per year

At $\mathrm{BMC}$, research is always in progress.

Learn more biomedcentral.com/submissions 